Analysis of the Fourth Spectrum of Tungsten (W IV)

This article has been downloaded from IOPscience. Please scroll down to see the full text article. 1985 Phys. Scr. 31173

(http://iopscience.iop.org/1402-4896/31/3/004)

View the table of contents for this issue, or go to the journal homepage for more

Download details:

IP Address: 161.111.22.141

The article was downloaded on 26/03/2013 at 10:05

Please note that terms and conditions apply. 


\title{
Analysis of the Fourth Spectrum of Tungsten (W IV)
}

\author{
L. Iglesias, V. Kaufman*, O. Garcia-Riquelme and F. R. Rico \\ Instituto de Optica, Serrano 121, Madrid, Spain
}

Received October 3, 1984; accepted October 12, 1984

\begin{abstract}
The spectrum of triply ionized tungsten (W IV) was produced in a sliding-spark discharge and recorded photographically on the NBS $10.7 \mathrm{~m}$ normal-incidence vacuum spectrograph in the $600-2600 \AA$ spectral region. The analysis has established all 37 levels of the $5 d^{3}, 5 d^{2} 6 s$ and $5 d 6 s^{2}$ even configurations and all 68 levels of the $5 d^{2} 6 p$ and $5 d 6 s 6 p$ odd confugurations. A total of 774 lines have been classified as transitions between these levels. Comparison of observed level values with those calculated in a least-squares fit shows an rms deviation of $\pm 50 \mathrm{~cm}^{-1}$ for the even configurations and $\pm 250 \mathrm{~cm}^{-1}$ for the odd ones.
\end{abstract}

\section{Introduction}

To our knowledge, no previous attempts have been made to analyze the spectrum of triply ionized tungsten. The ground configuration of this ion is $5 d^{3}$ and the other low, even configurations are $5 d^{2} 6 s$ and $5 d 6 s^{2}$. The lowest odd configurations are $5 d^{2} 6 p$ and $5 d 6 s 6 p$. In the analysis of a spectrum, one usually makes use of information available in isoelectronic and/ or isoionic spectra. The adjacent isoelectronic spectra, Ta III and $\operatorname{Re} \mathrm{V}$, are completely unknown. Among the fourth spectra of the platinum group, only the analysis of $\mathrm{Ta}[1]$, with a ground configuration of $5 d^{2}$, could provide relevant information.

\section{Observations}

A sliding-spark discharge with a quartz spacer was used to produce tungsten spectra. Peak currents of approximately 50 , 200 and $500 \mathrm{~A}$ gave excellent separation of W III, W IV and W V. It was found necessary to use He at approximately 20 Torr in order to maintain the discharge. A water-cooled copper hollow cathode, containing small pieces of germanium and silicon, was operated at $500 \mathrm{~mA}$ with $\mathrm{He}$ at 2 Torr to produce lines of $\mathrm{Cu}$ II, Ge I, Ge II, Si I and Si II which were used as standards [2]. The spectra were recorded photographically between 600 and $2600 \AA$ on the $10.7 \mathrm{~m}$ normal-incidence vacuum spectrograph at NBS. The estimated uncertainty in the measurements is $\pm 0.005 \AA$.

Approximately 1000 lines were identified as belonging to W IV and over 4000 as W III. An analysis of the W III data is underway. Analysis of those lines with W IV discharge characteristics has led to the classification of 774 lines. Seventeen of these are doubly classified, giving 791 transitions between the 37 even and 68 odd levels that were identified. Table I gives the wavelength, intensity, wavenumber, difference between the observed and calculated wavelengths, and classification of each of these lines.

\footnotetext{
* National Bureau of Standards, Washington, DC. Atomic \& Plasma Radiation Division.
}

\section{Analysis}

All of the predicted levels of the $5 d^{3}, 5 d^{2} 6 s, 5 d 6 s^{2}, 5 d^{2} 6 p$ and $5 d 6 s 6 p$ configurations have been determined. Hartree-Fock (HF) calculations were performed for the Slater and spin-orbit integrals of each of the configurations and for the relevant configuration interaction parameters. Comparison of the level structures predicted by parametric calculations and the repeating intervals found among the strong lines enabled the analysis to proceed. Tables II and III give the relevant information about the even and odd levels of triply ionized tungsten. Included for each level in the tables are the configuration, term, $J$ value, the level value and its uncertainty and the number of observed transitions to or from the level. The notation used to distinguish the various levels (e.g. $a, b, c, \ldots z, y, x, \ldots$ ) follows the recommendation of Russell et al [3]. The " 1 " and " 2 " in the terms $a^{2} D 2$ and $b^{2} D 1$ refer to the seniority numbers of the terms and are designated in the notation of Nielson and Koster [4].

Figure 1 shows the energy range occupied by all of the presently known configurations. The only overlap between the $5 d^{3}$ and the $5 d^{2} 6 s$ even configurations occurs between the highest term of $5 d^{3},{ }^{2} D 1$, and the lowest term of $5 d^{2} 6 s,{ }^{4} \mathrm{~F}$. This can be seen more clearly in Figure 2 which shows the energy levels of the three even configurations connected in $L S$ coupling terms.

The radial parameters derived by least-squares fitting (LSF) of the calculated to the experimental levels are given in Tables IV and $\mathrm{V}$ for the even and odd configurations, respectively. The table also includes the HF values and the LSF to HF ratios. These ratios are very similar to those found for triply ionized tantalum [1]. Table VI shows this similarity.

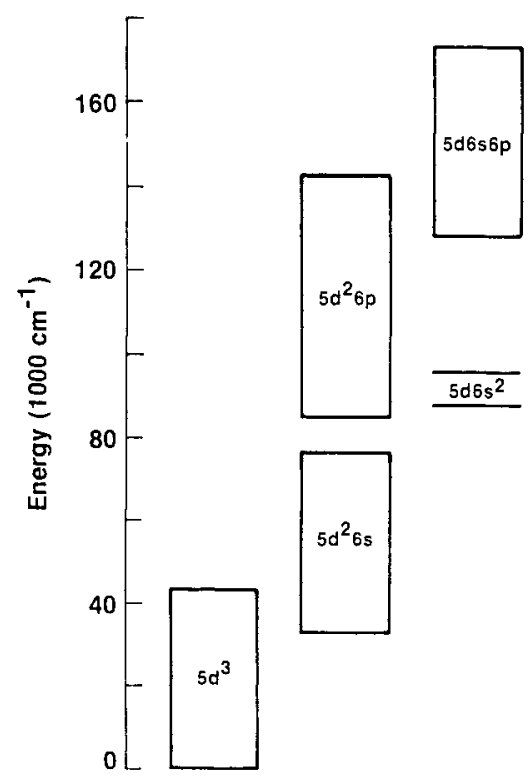

Fig. 1. Schematic diagram of the low configurations of W IV. 


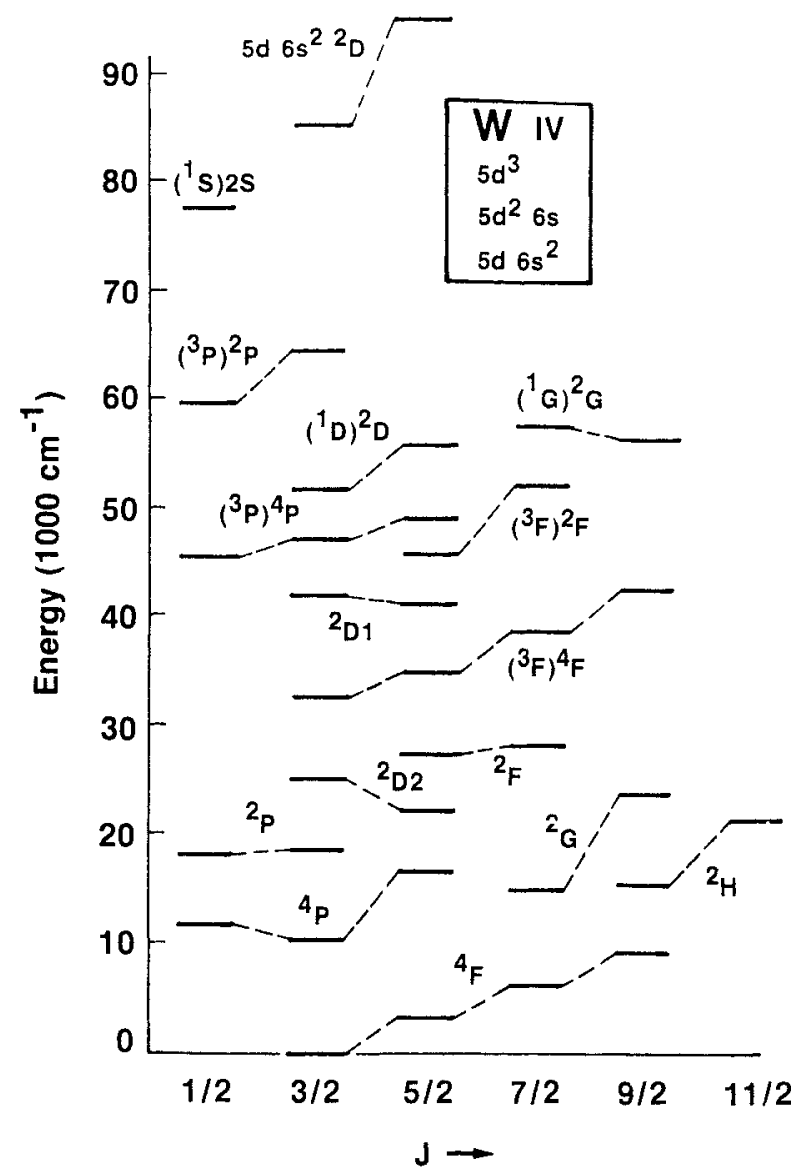

Fig. 2. Even levels of W IV. The $5 d^{2} 6 s$ terms include the $5 d^{2}$ parent term.

The even configurations, $5 d^{3}$ and $5 d^{2} 6 s$, show good $L S$ coupling with about $75 \%$ overall purity. Except for the $5 d^{3}$ level at $18519 \mathrm{~cm}^{-1}$ and the $5 d^{2} 6 s$ level at $46087 \mathrm{~cm}^{-1}$, all of the even levels have been given $L S$ names corresponding to the largest eigenvector component. Table II includes the difference between the observed level value and that calculated in the least-squares fit, o-c, and the leading eigenvector percentage(s) in $L S$ coupling. The mean deviation between the calculated and observed even levels is $\pm 50 \mathrm{~cm}^{-1}$.

The only overlap between the two odd configurations occurs between the $5 d^{2}\left({ }^{1} S\right) 6 p{ }^{2} P$ and the $5 d\left({ }^{2} D\right) 6 s 6 p\left({ }^{3} P\right){ }^{4} F$ and ${ }^{4} D$ terms. In calculating the odd configurations, several coupling schemes were considered. Neither $L S$ nor $J_{1} j$ coupling are pure, in $5 d^{2} 6 p$ the mean purity being $49 \%$ and $63 \%$ respectively. $L S$ designations for these levels have been chosen for simplicity but many of the levels show large eigenvector mixtures in this coupling. The names given to the levels of $J=3 / 2100449$, of $J=5 / 298691$ and 115317 , of $J=7 / 2106730,108872$ and 111515 , and of $J=9 / 2104263$ are not those of the largest eigenvector components. The compositions are very sensitive to small variations in the parameter values.

In the $5 d 6 s 6 p$ configuration, only the levels $J=3 / 2157726$ and $J=5 / 2149758$ have not been given the names of the largest eigenvector components. This configuration has an $S-L$ coupling purity of about $69 \%$. Table III includes the difference between the observed and calculated value and the leading $L S$ percentage(s) for each of the odd levels. The mean deviation between the calculated and observed odd levels is $\pm 250 \mathrm{~cm}^{-1}$ with configuration interaction included in the calculation.

The exchange integral, $G^{1}(s p)$, is very large emphasizing that the coupling between the $6 s$ and $6 p$ electrons is very strong. The best description of the $5 d 6 s 6 p$ configuration is then given by $5 d\left({ }^{2} D\right) 6 s 6 p\left({ }^{1,3} P\right)$. The strong $L S$ character of the intermediate ${ }^{1} P$ and ${ }^{3} P$ terms was noticed by Shenstone [5] in his analysis of $\mathrm{Cu}$ I. Martin and Sugar [6] found that this was also true for $\mathrm{Zn} \mathrm{II,} \mathrm{Ag} \mathrm{I}$ and $\mathrm{Cd}$ II. Johansson [7] also mentions this for the $3 d^{5} 4 s 4 p$ configuration in Fe II. This appears to be confirmed in W IV $5 d 6 s 6 p$ by observed intensity relations shown in Table VII.

\section{References}

1. Meijer, F. G., and Metsch, B. C., Physica 94C, 259 (1978)

2. Kaufman, V., and Edlén, B., J. Phys. Chem. Ref. Data 3, 825 (1974).

3. Russell, H. N., Shenstone, A. G., and Turner, L. A., Phys. Rev. 33, 900 (1929).

4. Nielson, C. W., and Koster, G. F., "Spectroscopic Coefficients for the $p^{n}, d^{n}$, and $f^{n}$ Configurations", $275 \mathrm{pp}$ (MIT Press, Cambridge, MA, 1963).

5. Shenstone, A. G., Phil. Trans. Roy. Soc. (London) A241, 37 (1948).

6. Martin, W. C., and Sugar, J., J. Opt. Soc. Am. 59, 1266 (1969).

7. Johansson, S., Physica Scripta 18, 217 (1978). 
Table I. Classified lines of W IV

\begin{tabular}{|c|c|c|c|c|c|c|c|c|c|}
\hline $\begin{array}{l}\text { Wavelength } \\
\text { (A) }\end{array}$ & Int. & $\begin{array}{l}\text { Wavenumber } \\
\left(\mathrm{cm}^{-1}\right)\end{array}$ & $\begin{array}{l}O-C \\
(\AA)\end{array}$ & Classification & $\begin{array}{l}\text { Wavelength } \\
(\AA)\end{array}$ & Int. & $\begin{array}{l}\text { Wavelength } \\
\left(\mathrm{cm}^{-1}\right)\end{array}$ & $\begin{array}{l}\mathrm{O}-\mathrm{C} \\
(\AA)\end{array}$ & Classification \\
\hline 2658.039 & $60 \mathrm{D}$ & 37610.53 & -.006 & $b^{4} P_{3 / 2}-z^{4} G_{s / 2}^{0}$ & 1960.318 & 10 & 510121 & .003 & $. b^{4} P_{3 / 2}-z^{4} D_{5 / 2}^{0}$ \\
\hline 2628.534 & $1 \mathrm{H}$ & 38032.68 & -.001 & $c^{2} D_{3 / 2}-z^{4} F_{3 / 2}^{0}$ & 1956.454 & 10 & 51112.9 & .000 & $b^{2} F_{5 / 2}-y^{2} D_{3 / 2}^{0}$ \\
\hline 2594.231 & 20 & 38535.54 & .000 & $b^{2} P_{3 / 2}-y^{4} D_{5 / 2}^{0 / 2}$ & 1951.640 & 60 & 51239.0 & -.011 & $b^{2} D_{5 / 2}-z^{4} F_{5 / 2}^{0}$ \\
\hline 2575.621 & 1 & 38813.96 & .001 & $a^{2} S_{1 / 2}-Z^{2} P_{3 / 2}^{0}$ & 1947.189 & 15 & 51356.1 & .001 & $c^{2} D_{3 / 2}-y^{4} D_{s / 2}^{0}$ \\
\hline 2574.190 & 200 & 38835.54 & -.001 & $b^{2} P_{1 / 2}-z^{4} D_{1 / 2}^{0}$ & 1020684 & 10 & &.- .002 & $b^{2} G_{9 / 2}^{3 / 2} \cdots y^{2} G_{7 / 2}^{0 / 2}$ \\
\hline 2550.102 & 900 & 39202.35 & .005 & $b^{4} P_{5 / 2}-z^{4} G_{5 / 2}^{0}$ & 1929.684 & 60 & 51822.0 & .000 & $c^{2} D_{3 / 2}-y^{4} D_{1 / 2}^{0}$ \\
\hline 2545.595 & 2 & 39271.76 & -.012 & $b^{2} P_{3 / 2}-z^{4} D_{3 / 2}^{0}$ & 1927.521 & 80 & 51880.1 & .004 & $b^{2} G_{7 / 2}-z^{2} H_{9 / 2}^{0}$ \\
\hline 2505.193 & 0 & 39905.06 & .000 & $d^{2} D_{3 / 2}-x^{2} F_{s / 2}^{0}$ & 1919.672 & 10 & 52092.2 & -.004 & $c^{2} D_{3 / 2}-z^{4} D_{3 / 2}^{0}$ \\
\hline 2498.157 & 200 & 40017.44 & .002 & $b^{2} F_{7 / 2}-z^{4} G_{7 / 2}^{0}$ & 1916.162 & 10 & 52187.7 & --.001 & $b^{4} P_{3 / 2}-z^{2} D_{3 / 2}^{0}$ \\
\hline 2491.942 & 5 & 40117.24 & .004 & $a^{2} S_{1 / 2}-z^{2} P_{1 / 2}^{0}$ & 1912.559 & $1 \mathrm{~B}$ & 52286.0 & -.001 & $b^{2} G_{7 / 2}-y^{2} F_{5 / 2}^{0}$ \\
\hline 2484.401 & 500 & 40239.00 & .000 & $b^{2} P_{1 / 2}-z^{2} D_{3 / 2}^{0}$ & 1907.116 & 50 & 52435.2 & -.001 & $b^{2} F_{3 / 2}-z^{4} F_{9 / 2}^{0}$ \\
\hline 2464.982 & 300 & 40555.98 & .002 & $b^{2} F_{s / 2}-z^{4} F_{3 / 2}^{0}$ & 1906.355 & 100 & 52456.1 & .011 & $c^{2} D_{5 / 2}-y^{2} G_{7 / 2}^{0}$ \\
\hline 2458.937 & 100 & 40655.68 & .003 & $b^{2} G_{7 / 2}-z^{4} G_{9 / 2}^{0}$ & 1901.225 & 900 & 52597.7 & -.003 & $b^{4} F_{3 / 2}-z^{4} G_{5 / 2}^{0}$ \\
\hline 2412.781 & 1 & 41433.35 & -.008 & $a^{2} S_{1 / 2}-x^{2} D_{3 / 2}^{0}$ & 1900.987 & 50 & 52604.2 & -.002 & $b^{4} P_{5 / 2}-z^{4} D_{5 / 2}^{0}$ \\
\hline 2400.391 & 200 & 41647.20 & .002 & $b^{2} P_{1 / 2}-z^{2} S_{1 / 2}^{0}$ & 1895.004 & 10 & 52770.3 & .001 & $b^{4} P_{3 / 2}-y^{2} D_{3 / 2}^{0}$ \\
\hline 2397.528 & 500 & 41696.93 & .006 & $b^{2} F_{7 / 2}-z^{4} F_{5 / 2}^{0}$ & 1888.687 & 200 & 52946.8 & -.002 & $b^{4} P_{1 / 2}^{3 / 2}-z^{4} D_{1 / 2}^{3 / 2}$ \\
\hline 2381.467 & 500 & 41978.12 & .005 & $b^{2} G_{9 / 2}-z^{4} G_{9 / 2}^{0}$ & 1885.217 & 10 & 53044.3 & -.002 & $b^{2} P_{3 / 2}^{1 / 2}-z^{2} P_{1 / 2}^{1 / 2}$ \\
\hline 2380.042 & 40 & 42003.25 & .008 & $b^{2} D_{s / 2}-z^{4} G_{5 / 2}^{0}$ & 1881.728 & 20 & 53142.6 & --.001 & $b^{2} G_{7 / 2}^{3 / 2}-y^{4} D_{7 / 2}^{1 / 2}$ \\
\hline 2376.076 & 500 & 42073.35 & .004 & $b^{2} P_{3 / 2}-z^{2} F_{5 / 2}^{0}$ & 1879.601 & 100 & 53202.8 & -.003 & $b^{2} G_{9 / 2}^{7 / 2}-z^{2} H_{9 / 2}^{0 / 2}$ \\
\hline 2364.676 & $10 \mathrm{H}$ & 42276.17 & .003 & $c^{2} D_{5 / 2}-z^{4} D_{5 / 2}^{0}$ & 1870.317 & 100 & 53466.9 & -.002 & $c^{2} D_{5 / 2}^{9 / 2}-z^{4} S_{3,2}^{0}$ \\
\hline 2343.137 & 500 & 42664.76 & .006 & $c^{2} D_{3 / 2}-z^{4} F_{5 / 2}^{0}$ & 1868.086 & 15 & 53530.7 & -.005 & $b^{2} F_{5,2}-z^{2} F_{7 / 2}^{0}$ \\
\hline 2300.701 & 100 & 43451.63 & .001 & $c^{2} D_{5 / 2}-z^{2} D_{3 / 2}^{0}$ & 1865.815 & 60 & 53595.9 & .000 & $b^{4} P_{3 / 2}-z^{2} S_{1 / 2}^{0}$ \\
\hline 2297.688 & 500 & 43508.61 & .003 & $b^{2} F_{5 / 2}^{3 / 2}-z^{4} G_{7 / 2}^{0}$ & 1858.182 & 800 & 53816.0 & -.002 & $b^{4} F_{7 / 2}-z^{4} G_{7 / 2}^{0}$ \\
\hline 2282.116 & 200 & 43805.46 & .003 & $b^{4} P_{5 / 2}-z^{4} F_{3 / 2}^{0}$ & 1855.997 & 60 & 53879.4 & .002 & $b^{2} F_{s / 2}-y^{4} D_{s / 2}^{0}$ \\
\hline 2276.850 & $10 \mathrm{H}$ & 43906.77 & .003 & $c^{2} D_{s / 2}-z^{4} F_{7 / 2}^{0}$ & 1855.213 & 60 & 53902.2 & .000 & $b^{2} F_{7 / 2}-z^{2} G_{7 / 2}^{0}$ \\
\hline 2270.245 & 80 & 44034.50 & -.006 & $c^{2} D_{5 / 2}-y^{2} D_{3 / 2}^{0}$ & 1854.391 & 50 & 53926.1 & .004 & $b^{2} F_{7 / 2}^{7 / 2}-z^{2} F_{s / 2}^{0^{\prime 2}}$ \\
\hline 2268.424 & $60 \mathrm{H}$ & 44069.84 & .003 & $a^{2} S_{1 / 2}-y^{2} P_{1 / 2}^{0}$ & 1843.839 & 2 & 54234.7 & .005 & $b^{4} P_{5 / 2}^{7 / 2}-z^{4} F_{7 / 2}^{0}$ \\
\hline 2259.630 & $10 \mathrm{H}$ & 44241.34 & .002 & $b^{2} P_{3 / 2}-y^{4} D_{3 / 2}^{0}$ & 1843.559 & 30 & 54242.9 & .001 & $c^{2} D_{5 / 2}^{5 / 2}-y^{2} F_{5 / 2}^{1 / 2}$ \\
\hline 2255.396 & 300 & 44324.38 & -.001 & $b^{2} P_{1 / 2}-z^{4} D_{3 / 2}^{0}$ & 1839.933 & 2 & 54349.8 & .015 & $b^{4} P_{1 / 2}^{5 / 2}-z^{2} D_{3 / 2}^{0 / 2}$ \\
\hline 2252.767 & 2 & 44376.10 & .001 & $b^{4} P_{1 / 2}-z^{4} F_{3 / 2}^{0}$ & 1839.514 & 100 & 54362.2 & .006 & $b^{4} P_{5 / 2}-y^{2} D_{3 / 2}^{0}$ \\
\hline 2246.748 & 200 & 44494.98 & .006 & $b^{2} G_{7 / 2}-z^{2} F_{7 / 2}^{0}$ & 1836.041 & 50 & 54465.0 & .003 & $b^{2} G_{9 / 2}-y^{4} D_{7 / 2}^{0}$ \\
\hline 2212.274 & 200 & 45188.28 & -.002 & $b^{2} F_{s / 2}-z^{4} F_{s / 2}^{0}$ & 1830.986 & 2 & 54615.4 & .004 & $b^{2} F_{5 / 2}-z^{4} D_{3 / 2}^{0}$ \\
\hline 2211.633 & 50 & 45201.37 & .000 & $b^{2} P_{3 / 2}^{0 / 2}-z^{4} S_{3 / 2}^{0 / 2}$ & 1824.848 & 300 & 54799.1 & .007 & $b^{4} F_{5 / 2}^{3 / 2}-z^{4} F_{3 / 2}^{0}$ \\
\hline 2174.297 & 200 & 45977.46 & .002 & $b^{2} P_{3 / 2}^{3 / 2}-y^{2} F_{5 / 2}^{0}$ & 1820.411 & $60 \mathrm{~B}$ & 54932.7 & .011 & $b^{4} P_{1 / 2}-y^{2} D_{3 / 2}^{0}$ \\
\hline 2170.234 & $30 \mathrm{H}$ & 46063.53 & -.001 & $d^{2} D_{5 / 2}-y^{4} F_{7 / 2}^{0}$ & 1801.947 & 500 & 55495.5 & .001 & $b^{4} F_{7 / 2}-z^{4} F_{5 / 2}^{0}$ \\
\hline 2166.095 & 40 & 46151.54 & .000 & $b^{2} D_{3 / 2}-z^{4} F_{3 / 2}^{0}$ & 1800.605 & 50 & 55536.9 & .002 & $b^{4} P_{3 / 2}^{7 / 2}-y^{4} D_{5 / 2}^{0}$ \\
\hline 2164.479 & 10 & 46186.00 & -.001 & $d^{2} D_{3 / 2}-x^{2} P_{1 / 2}^{0}$ & 1793.715 & 2 & 55750.2 & .001 & $a^{2} S_{1 / 2}-x^{2} P_{1 / 2}^{0}$ \\
\hline 2163.819 & 900 & 46200.08 & -.003 & $b^{2} F_{7 / 2}-z^{4} G_{9 / 2}^{0}$ & 1791.987 & 1000 & 55804.0 & -.003 & $b^{4} F_{9 / 2}-z^{4} G_{9 / 2}^{0}$ \\
\hline 2161.004 & $10 \mathrm{~B}$ & 46260.26 & .000 & $b^{4} F_{9 / 2}-z^{4} G_{5 / 2}^{0}$ & 1785.626 & 5 & 56002.8 & .002 & $b^{4} P_{3 / 2}-y^{4} D_{1 / 2}^{0}$ \\
\hline 2152.084 & 100 & 46451.98 & .005 & $c^{2} D_{s / 2}-z^{2} F_{7 / 2}^{0}$ & 1784.319 & 2 & 56043.8 & -.002 & $b^{2} F_{7 / 2}-y^{2} G_{7 / 2}^{0}$ \\
\hline 2145.094 & 30 & 46603.33 & -.001 & $c^{2} D_{3 / 2}-z^{4} D_{1 / 2}^{0}$ & 1777.052 & 80 & 56273.0 & .001 & $b^{4} P_{3 / 2}-z^{4} D_{3 / 2}^{0}$ \\
\hline 2144.951 & 60 & 46606.43 & .001 & $b^{2} D_{5 / 2}-z^{4} F_{3 / 2}^{0}$ & 1767.391 & 20 & 56580.6 & .000 & $b^{2} D_{5 / 2}-z^{2} D_{3 / 2}^{0}$ \\
\hline 2137.992 & 500 & 46758.12 & .002 & $b^{4} P_{5 / 2}-z^{4} G_{7 / 2}^{0}$ & 1761.182 & 60 & 56780.0 & .001 & $b^{4} P_{s / 2}-z^{2} F_{7 / 2}^{0}$ \\
\hline 2134.649 & 300 & 46831.33 & .001 & $c^{2} D_{3 / 2}^{2}-z^{4} D_{5 / 2}^{0 / 2}$ & 1754.475 & 25 & 56997.1 & -.009 & $b^{2} P_{3 / 2}-y^{2} P_{1 / 2}^{0}$ \\
\hline 2133.990 & 10 & 46845.80 & -.003 & $b^{4} P_{3 / 2}-z^{4} F_{5 / 2}^{0}$ & 1752.833 & 100 & 57050.5 & -.003 & $b^{2} G_{7 / 2}-z^{2} G_{9 / 2}^{0}$ \\
\hline 2131.934 & 5 & 46890.97 & -.005 & $b^{2} G_{7 / 2}-z^{4} F_{9 / 2}^{0}$ & 1752.476 & 2 & 57062.1 & -.006 & $c^{2} D_{3 / 2}-y^{4} D_{3 / 2}^{0}$ \\
\hline 2105.550 & 20 & 47478.47 & -.001 & $d^{2} D_{s / 2}-x^{2} P_{3 / 2}^{0}$ & 1751.365 & 300 & 57098.3 & -.011 & $b^{4} F_{9 / 2}-z^{4} F_{7 / 2}^{0}$ \\
\hline 2104.861 & $900 \mathrm{~B}$ & 47494.01 & .005 & $b^{2} F_{7 / 2}-z^{4} F_{7 / 2}^{0}$ & 1750.422 & 50 & 57129.1 & -.004 & $b^{4} P_{5 / 2}-y^{4} D_{5 / 2}^{0}$ \\
\hline 2102.957 & 40 & 47537.01 & .000 & $c^{2} D_{5 / 2}-z^{4} D_{3 / 2}^{0}$ & 1749.364 & 1 & 57163.6 & -.010 & $b^{2} D_{5 / 2}-y^{2} D_{3 / 2}^{0}$ \\
\hline 2082.382 & 100 & 48006.64 & .006 & $c^{2} D_{3 / 2}-z^{2} D_{3 / 2}^{0}$ & $\begin{array}{l}1749.004 \\
1748.227\end{array}$ & 200 & 57200.8 & -.005 & $b^{4} F_{3 / 2}^{5 / 2}-z^{4} F_{3 / 2}^{0}$ \\
\hline 2074.877 & 30 & 48180.26 & -.001 & $b^{2} P_{3 / 2}-z^{4} P_{1 ; 2}^{0}$ & 1748.082 & 10 & 57205.6 & -.004 & $c^{2} D_{5 / 2}-y^{2} D_{5,2}^{0}$ \\
\hline 2073.454 & 500 & 48213.32 & .001 & $b^{2} G_{9 / 2}-z^{4} F_{9 / 2}^{0}$ & 1743.593 & 150 & 57352.8 & -.015 & $a^{2} F_{5 / 2}-z^{4} G_{5 / 2}^{0}$ \\
\hline 2067.260 & 300 & 48357.76 & .004 & $b^{2} G_{7 / 2}-z^{2} G_{7 / 2}^{0}$ & 1742.358 & 30 & 57393.5 & -.004 & $b^{2} F_{5 / 2}^{5 / 2}-z^{2} G_{7 / 2}^{0}$ \\
\hline 2066.227 & $500 \mathrm{D}$ & 48381.94 & -.003 & $b^{2} G_{7 / 2}-z^{2} F_{5 / 2}^{0}$ & 1741.625 & 150 & 57417.6 & -.009 & $b^{2} F_{5 / 2}-z^{2} F_{5 / 2}^{0}$ \\
\hline 2063.848 & 500 & 48437.70 & .001 & $b^{4} P_{5 / 2}-z^{4} F_{5 / 2}^{0}$ & 1741.415 & 50 & 57424.6 & -.001 & $b^{2} F_{7 / 2}-z^{2} H_{9 / 2}^{0}$ \\
\hline 2057.400 & 3 & 48589.49 & .001 & $c^{2} D_{3 / 2}-y^{2} D_{3 / 2}^{0}$ & $\begin{array}{l}1741.413 \\
1731.969\end{array}$ & 10 & 57737.8 & .004 & $b^{2} G_{7 / 2}^{7 / 2}-z^{2} D_{7 / 2}^{0}$ \\
\hline 2046.340 & $50 \mathrm{H}$ & 48852.06 & .003 & $b^{2} P_{3 / 2}-z^{4} P_{3 / 2}^{0}$ & 1731.535 & 1000 & 57752.2 & -.009 & $b^{4} F_{s / 2}-z^{4} G_{7 / 2}^{0}$ \\
\hline 2042.665 & $30 \mathrm{H}$ & 48939.94 & .002 & $b^{2} P_{3 / 2}-y^{2} D_{5 / 2}^{0}$ & 1728.156 & 50 & 57865.1 & -.005 & $b^{4} P_{5 / 2}-z^{4} D_{3 / 2}^{0}$ \\
\hline 2023.020 & 300 & 49415.12 & -.004 & $c^{2} D_{3 / 2}-z^{2} S_{1 / 2}^{0}$ & 1721.259 & 15 & 58097.0 & .000 & $b^{2} P_{1 / 2}-z^{2} P_{1 / 2}^{0}$ \\
\hline 2017.145 & 300 & 49559.02 & .003 & $b^{2} D_{5 / 2}-z^{4} G_{7 / 2}^{0}$ & 1719.231 & 40 & 58165.5 & -.003 & $b^{4} P_{1 / 2}-y^{4} D_{1 / 2}^{0}$ \\
\hline 2014.618 & 100 & 49621.2 & .005 & $b^{4} F_{9 / 2}-z^{4} G_{7 / 2}^{0}$ & 1713.126 & 50 & 58372.8 & .002 & $b^{2} G_{9 / 2}-z^{2} G_{9 / 2}^{0}$ \\
\hline 2012.216 & 200 & 49680.4 & -.002 & $b^{2} G_{9 / 2}^{3 / 2}-z^{2} G_{7 / 2}^{0}$ & 1711.285 & 25 & 58435.6 & -.001 & $b^{4} P_{1 / 2}-z^{4} D_{3 / 2}^{0}$ \\
\hline 1998.421 & 100 & 50039.5 & -.005 & $b^{2} F_{7 / 2}-z^{2} F_{7 / 2}^{0}$ & 1708.351 & 50 & 58536.0 & .000 & $b^{2} P_{3 / 2}-x^{2} D_{5 / 2}^{0}$ \\
\hline 1992.181 & 900 & 50196.2 & -.002 & $b^{4} F_{5 / 2}-z^{4} G_{5 / 2}^{0}$ & 1703.957 & 50 & 58686.9 & .000 & $b^{2} F_{7 / 2}-y^{4} D_{7 / 2}^{0}$ \\
\hline 1980.220 & 100 & 50499.4 & -.001 & $b^{2} G_{7 / 2}-y^{2} G_{7 / 2}^{0}$ & 1700.742 & 200 & 58797.9 & .006 & $c^{2} D_{3 / 2}-y^{2} F_{5 / 2}^{0}$ \\
\hline 1979.017 & 100 & 50530.1 & .001 & $b^{2} F_{5 / 2}-z^{2} D_{3 / 2}^{0}$ & 1697.732 & 35 & 58902.1 & .002 & $c^{2} D_{5 / 2}-z^{4} P_{5 / 2}^{0}$ \\
\hline 1961.352 & 2 & 50985.2 & .003 & $b^{2} F_{5 / 2}-z^{4} F_{7 / 2}^{0}$ & 1693.183 & 20 & 59060.4 & .001 & $b^{2} G_{9 / 2}-z^{4} D_{7 / 2}^{0}$ \\
\hline
\end{tabular}

Table I. Continued 
Table I. Continued

\begin{tabular}{|c|c|c|c|c|c|c|c|c|c|}
\hline $\begin{array}{l}\text { Wavelength } \\
\text { (A) }\end{array}$ & Int. & $\begin{array}{l}\text { Wavenumber } \\
\left(\mathrm{cm}^{-1}\right)\end{array}$ & $\begin{array}{l}\mathrm{O}-\mathrm{C} \\
(\AA)\end{array}$ & Classification & $\begin{array}{l}\text { Wavelength } \\
\text { (A) }\end{array}$ & Int. & $\begin{array}{l}\text { Wavenumber } \\
\left(\mathrm{cm}^{-1}\right)\end{array}$ & $\begin{array}{l}\mathrm{O}-\mathrm{C} \\
(\AA)\end{array}$ & Classification \\
\hline 1683.141 & 50 & 59412.7 & .006 & $b^{2} P_{1 / 2}-x^{2} D_{3 / 2}^{0}$ & 1497.604 & 300 & 66773.3 & .001 & $b^{2} F_{5 / 2}-z^{4} D_{7 / 2}^{0}$ \\
\hline 1682.611 & 150 & 59431.4 & .001 & $b^{4} F_{5 / 2}-z^{4} F_{5 / 2}^{0}$ & 1496.973 & 50 & 66801.5 & -.002 & $c^{2} D_{\mathrm{s} / 2} \cdots x^{2} D_{\mathrm{s} / 2}^{0}$ \\
\hline 1679.688 & 50 & 59534.9 & .001 & $b^{2} F_{5 / 2}-y^{2} G_{7 / 2}^{0}$ & 1495.704 & 250 & 66858.1 & .000 & $b^{2} G_{9 / 2}-x^{2} F_{7 / 2}^{\circ}$ \\
\hline 1678.389 & 25 & 59580.9 & .002 & $b^{2} D_{5 / 2}-z^{2} F_{7 / 2}^{0}$ & 1495.532 & 100 & 66865.8 & -.001 & $b^{2} F_{7 / 2}-y^{2} G_{9 / 2}^{0}$ \\
\hline 1678.274 & 10 & 59585.0 & .008 & $b^{2} F_{5 / 2}-y^{4} D_{3 / 2}^{0}$ & $\begin{array}{l}1495.532 \\
1491.903\end{array}$ & 300 & 67028.5 & -.003 & $b^{4} F_{9 / 2}^{7 / 2}-z^{2} H_{9 / 2}^{0}$ \\
\hline 1676.638 & 100 & 59643.2 & .002 & $b^{4} F_{9 / 2}-z^{2} F_{7 / 2}^{0}$ & 1490.651 & 25 & 67084.8 & .001 & $b^{2} F_{5 / 2}-z^{2} P_{3 / 2}^{0}$ \\
\hline 1676.112 & 50 & 59661.9 & .004 & $b^{4} F_{7 / 2}-z^{4} D_{5 / 2}^{0}$ & $\begin{array}{l}1490.031 \\
1489.721\end{array}$ & 35 & 67126.7 & .001 & $a^{2} D_{5 / 2}^{s / 2}-z^{4} F_{3 / 2}^{0}$ \\
\hline 1675.182 & 25 & 59695.0 & -.003 & $c^{2} D_{5 / 2}-z^{4} D_{7 / 2}^{0}$ & 1488.653 & 250 & 67174.8 & -.001 & $b^{4} F_{3 / 2}-z^{2} D_{3 / 2}^{0}$ \\
\hline 1666.707 & 500 & 59998.5 & .000 & $b^{4} F_{7 / 2}^{5 / 2}-z^{4} G_{9 / 2}^{0}$ & 1487.682 & 100 & 67218.7 & -.001 & $c^{2} D_{3 / 2}-z^{2} D_{5 / 2}^{0}$ \\
\hline 1651.658 & 50 & 60545.2 & .002 & $b^{2} F_{5 / 2}^{1 / 2}-z^{4} S_{3 / 2}^{0}$ & 1481.644 & 25 & 67492.6 & .002 & $c^{2} D_{5 / 2}-x^{2} F_{7 / 2}^{0}$ \\
\hline 1648.999 & 200 & 60642.9 & .000 & $b^{4} P_{5 / 2}^{5 / 2}-z^{2} G_{7 / 2}^{0}$ & 1480.747 & 50 & 67533.5 & -.002 & $b^{4} P_{5 / 2}^{0 / 2}-y^{2} D_{5 / 2}^{0}$ \\
\hline 1648.348 & 30 & 60666.8 & .001 & $b^{4} P_{5 / 2}^{5 / 2}-z^{2} F_{5 / 2}^{0}$ & 1478.455 & 350 & 67638.2 & .000 & $b^{4} P_{3 / 2}-z^{4} P_{5 / 2}^{0}$ \\
\hline 1647.264 & 45 & 60706.7 & -.006 & $b^{2} G_{7 / 2}-z^{2} D_{5 / 2}^{0}$ & 1477.089 & 250 & 67700.7 & -.001 & $b^{4} F_{7 / 2}-z^{2} G_{7 / 2}^{0}$ \\
\hline 1644.933 & 100 & 60792.7 & -.001 & $b^{2} F_{7 / 2}-y^{2} D_{5 / 2}^{0}$ & 1476.570 & 100 & 67724.5 & .003 & $b^{4} F_{7 / 2}^{7 / 2}-z^{2} F_{5 / 2}^{0^{\prime}}$ \\
\hline 1632.849 & 40 & 61242.6 & .004 & $b^{4} P_{3 / 2}-y^{4} D_{3 / 2}^{0}$ & 1475.851 & 250 & 67757.5 & .000 & $b^{4} F_{3 / 2}-y^{2} D_{3 / 2}^{0 / 2}$ \\
\hline 1631.516 & 200 & 61292.7 & -.001 & $b^{4} F_{7 / 2}-z^{4} F_{7 / 2}^{0}$ & 1475.490 & 350 & 67774.1 & -.005 & $b^{4} F_{5 / 2}^{3 / 2}-z^{2} F_{7 / 2}^{0}$ \\
\hline 1630.746 & 250 & 61321.6 & -.005 & $b^{2} F_{s / 2}-y^{2} F_{s / 2}^{0}$ & 1470.235 & 200 & 68016.3 & -.004 & $b^{4} P_{1 / 2}^{3 / 2}-z^{2} P_{3 / 2}^{0}$ \\
\hline & & & & $b^{2} G_{7 / 2}-y^{2} G_{9 / 2}^{0}$ & 1468.424 & 350 & 68100.2 & -.003 & $a^{4} P_{s / 2}-z^{4} G_{s / 2}^{0}$ \\
\hline 1628.773 & 100 & 61395.9 & -.005 & $b^{2} P_{3 / 2}-y^{2} P_{3 / 2}^{0}$ & 1467.939 & 200 & 68122.7 & .000 & $b^{4} F_{5 / 2}-y^{4} D_{5 / 2}^{0}$ \\
\hline 1614.060 & 50 & 61955.6 & -.004 & $a^{2} F_{s / 2}-z^{4} F_{3 / 2}^{0}$ & 1464.327 & 150 & 68290.8 & .000 & $b^{4} F_{9 / 2}-y^{4} D_{7 / 2}^{0}$ \\
\hline 1611.893 & 250 & 62038.9 & .004 & $b^{4} F_{9 / 2}-z^{4} F_{9 / 2}^{0}$ & 1458.088 & 100 & 68583.0 & .001 & $b^{4} F_{3 / 2}-z^{2} S_{1 / 2}^{0}$ \\
\hline 1611.613 & 35 & 62049.6 & -.001 & $b^{2} P_{1 / 2}-y^{2} P_{1 / 2}^{0}$ & 1455.503 & 200 & 68704.8 & -.001 & $b^{2} G_{7 / 2}-x^{2} F_{5 / 2}^{0}$ \\
\hline 1608.282 & 100 & 62178.2 & -.001 & $b^{2} F_{5 / 2}-y^{4} D_{7 / 2}^{0}$ & 1453.156 & 30 & 68815.7 & .002 & $b^{2} F_{7 / 2}-y^{2} F_{7 / 2}^{0}$ \\
\hline 1607.644 & 40 & 62202.8 & -.001 & $b^{4} P_{3 / 2}-z^{4} S_{3 / 2}^{0}$ & $\begin{array}{l}1453.130 \\
1452.249\end{array}$ & 50 & 68858.7 & .001 & $b^{4} F_{5 / 2}^{1 / 2}-z^{4} D_{3 / 2}^{0}$ \\
\hline 1602.657 & 30 & 62396.4 & -.003 & $b^{2} P_{3 / 2}-x^{2} F_{5 / 2}^{0}$ & 1444.456 & 100 & 69230.2 & -.001 & $b^{4} P_{5 / 2}^{5 / 2}-z^{4} P_{5 / 2}^{0}$ \\
\hline 1599.394 & 100 & 62523.7 & -.002 & $a^{2} D_{5 / 2}-z^{4} G_{5 / 2}^{0}$ & 1431.797 & 100 & 69842.3 & -.001 & $b^{4} F_{7 / 2}-y^{2} G_{7 / 2}^{0}$ \\
\hline 1579.573 & 300 & 62594.9 & -.007 & $b^{2} F_{1 / 2}-z^{2} G_{9 / 2}^{0}$ & 1428.101 & 150 & 70023.1 & -.004 & $b^{4} P_{s / 2}-z^{4} D_{7 / 2}^{0}$ \\
\hline 1596.317 & 200 & 62644.2 & -.005 & $b^{2} G_{9 / 2}-y^{2} G_{9 / 2}^{0}$ & 1427.975 & 50 & 70029.2 & .004 & $a^{2} G_{7 / 2}-z^{4} G_{5 / 2}^{0}$ \\
\hline 1595.825 & 100 & 62663.5 & .000 & $c^{2} D_{5 / 2}-z^{2} D_{s / 2}^{0}$ & 1427.640 & 15 & 70045.7 & .000 & $b^{4} P_{3 / 2}-z^{2} P_{1 / 2}^{0}$ \\
\hline 1592.746 & 200 & 62784.6 & -.006 & $b^{4} P_{5 / 2}^{3 / 2}-y^{2} G_{7 / 2}^{0 / 2}$ & 1426.951 & 50 & 70079.5 & -.003 & $a^{2} D_{5 / 2}-z^{4} G_{7 / 2}^{0}$ \\
\hline 1591.471 & 150 & 62834.9 & -.004 & $b^{4} P_{5 / 2}-y^{4} D_{3 / 2}^{0}$ & & & & .000 & $b^{4} P_{5 / 2}-z^{2} P_{3 / 2}^{0}$ \\
\hline 1586.980 & 40 & 63012.8 & .003 & $b^{2} D_{3 / 2}-z^{2} F_{5 / 2}^{0}$ & 1421.781 & 3 & 10334.5 & .001 & $b^{2} D_{5 / 2}-y^{2} D_{5 / 2}^{0}$ \\
\hline 1580.490 & 100 & 63271.5 & .000 & $b^{2} G_{7 / 2}-y^{2} F_{7 / 2}^{0}$ & 1420.678 & 100 & 70388.9 & -.005 & $b^{2} F_{7 / 2}-x^{2} D_{5 / 2}^{0}$ \\
\hline 1580.219 & 50 & 63282.4 & -.004 & $b^{2} F_{7 / 2}-z^{4} D_{7 / 2}^{0}$ & 1417.953 & 100 & 70524.2 & -.002 & $b^{4} F_{3 / 2}-y^{4} D_{s / 2}^{0}$ \\
\hline 1577.148 & 50 & 63405.6 & -.005 & $b^{4} P_{1 / 2}-y^{4} D_{3 / 2}^{0}$ & 1416.102 & 100 & 70616.4 & -.005 & $a^{2} F_{7 / 2}-z^{4} G_{9 / 2}^{0}$ \\
\hline 1575.857 & 20 & 63457.5 & -.006 & $c^{2} D_{3 / 2}-z^{4} P_{5 / 2}^{0}$ & 1415.192 & 100 & 70661.8 & -.002 & $c^{2} D_{5 / 2}-x^{2} F_{\mathrm{s} / 2}^{0}$ \\
\hline 1575.600 & 40 & 63467.9 & -.002 & $b^{2} D_{5 / 2}-z^{2} F_{5 / 2}^{0}$ & 1413.340 & 100 & 70754.4 & -.005 & $a^{2} F_{s / 2}-z^{4} D_{s / 2}^{0}$ \\
\hline 1572.377 & 300 & 63598.0 & .000 & $b^{4} F_{s / 2}-z^{4} D_{s / 2}^{0}$ & 1410.338 & $200 \mathrm{D}$ & 70905.0 & -.001 & $b^{4} P_{1 / 2}-z^{2} P_{3 / 2}^{0}$ \\
\hline 1566.463 & 100 & 63838.1 & -.005 & $b^{4} F_{7 / 2}-z^{2} F_{7 / 2}^{0}$ & 1408.649 & $500 \mathrm{H}$ & 70990.0 & -.001 & $b^{4} F_{3 / 2}-y^{4} D_{1 / 2}^{0}$ \\
\hline 1557.952 & 100 & 64186.8 & -.001 & $b^{4} F_{7 / 2}-y^{4} D_{5 / 2}^{0}$ & 1406.864 & 50 & 71080.1 & -.002 & $b^{2} F_{7 / 2}-x^{2} F_{7 / 2}^{0}$ \\
\hline 1555.596 & 50 & 64284.0 & -.003 & $b^{2} F_{5 / 2}-y^{2} D_{5 / 2}^{0}$ & 1404.036 & $200 \mathrm{H}$ & 71223.2 & -.004 & $b^{4} F_{7 / 2}-z^{2} H_{9 / 2}^{0}$ \\
\hline 1551.987 & 150 & 64433.5 & .001 & $a^{2} F_{7 / 2}-z^{4} G_{7 / 2}^{0}$ & 1403.312 & 40 & 71260.0 & .003 & $b^{4} F_{3 / 2}-z^{4} D_{3 / 2}^{0}$ \\
\hline 1548.905 & 150 & 64561.7 & -.007 & $c^{2} D_{3 / 2}-z^{2} P_{3 / 2}^{0}$ & 1401.413 & 20 & 71356.6 & .000 & $c^{2} D_{3 / 2}-x^{2} D_{5 / 2}^{0}$ \\
\hline 1548.682 & 50 & 64571.0 & -.003 & $b^{4} P_{5 / 2}-y^{2} F_{5 / 2}^{0}$ & 1401.314 & 200 & 71361.6 & -.001 & $b^{4} P_{3 / 2}-x^{2} D_{3 / 2}^{0}$ \\
\hline 1548.089 & 50 & 64595.8 & -.004 & $a^{2} S_{1 / 2}-x^{2} P_{3 / 2}^{0}$ & 1401.069 & 100 & 71374.1 & .000 & $a^{2} D_{3 / 2}-z^{4} F_{3 / 2}^{0}$ \\
\hline 1543.841 & 4 & 64773.5 & -.003 & $b^{4} F_{5 / 2}-z^{2} D_{3 / 2}^{0}$ & 1400.568 & 600 & 71399.6 & -.002 & $b^{4} P_{3 / 2}-z^{2} D_{5 / 2}^{0}$ \\
\hline 1542.151 & 10 & 64844.5 & -.002 & $b^{2} G_{7 / 2}-x^{2} D_{5 / 2}^{0}$ & 1398.036 & $200 \mathrm{H}$ & 71528.9 & -.012 & $a^{2} P_{1 / 2}-z^{4} F_{3 / 2}^{0}$ \\
\hline 1534.172 & 100 & 65181.7 & -.002 & $b{ }^{4} P_{3 / 2}-z^{4} P_{1 / 2}^{0}$ & 1397.115 & 10 & 71576.1 & .002 & $b^{2} D_{3 / 2}-z^{4} P_{s / 2}^{0}$ \\
\hline & & & -.006 & $c^{2} D_{5 / 2}-y^{2} F_{7 / 2}^{0}$ & 1396.088 & 200 & 71628.7 & .000 & $b^{4} F_{7 / 2}-y^{2} F_{5 / 2}^{0}$ \\
\hline 1533.066 & 450 & 65228.8 & -.004 & $b^{4} F_{5 / 2}-z^{4} F_{7 / 2}^{0}$ & 1395.935 & 200 & 71636.6 & .001 & $b^{4} F_{s / 2}-z^{2} G_{7 / 2}^{0}$ \\
\hline 1530.081 & 100 & 65356.0 & .003 & $b^{4} F_{5 / 2}^{5 / 2}-y^{2} D_{3 / 2}^{0}$ & 1395.466 & $400 \mathrm{H}$ & 71660.6 & -.001 & $b^{4} F_{s / 2}-z^{2} F_{s / 2}^{0}$ \\
\hline 1528.405 & 50 & 65427.7 & -.002 & $b^{4} P_{5 / 2}^{5 / 2}-y^{4} D_{7 / 2}^{0}$ & 1393.553 & $100 \mathrm{~B}$ & 71759.0 & -.002 & $a^{2} D_{5 / 2}-z^{4} F_{5 / 2}^{0}$ \\
\hline 1525.885 & 150 & 65535.7 & -.002 & $b^{2} G_{7 / 2}^{0}-x^{2} F_{7 / 2}^{0}$ & 1390.611 & $400 \mathrm{D}$ & 71910.8 & -.012 & $a^{2} F_{7 / 2}-z^{4} F_{7 / 2}^{0}$ \\
\hline 1523.561 & 30 & 65635.7 & .001 & $b^{2} D_{5 / 2}-y^{4} D_{3 / 2}^{0}$ & & 100 & 71929.6 & & \\
\hline 1523.285 & 100 & 65647.6 & -.001 & $b^{4} F_{9 / 2}-y^{2} G_{7 / 2}^{0}$ & 1388.278 & $80 \mathrm{D}$ & 72031.7 & -.011 & $b^{2} D_{5 / 2}^{5 / 2}-z^{4} P_{5 / 2}^{0 / 2}$ \\
\hline 1520.418 & 150 & 65771.4 & -.002 & $b^{4} F_{3 / 2}-z^{4} D_{1 / 2}^{0}$ & 1385.070 & 400 & 72198.5 & .000 & $b^{4} F_{9 / 2}^{5 / 2}-z^{2} G_{9 / 2}^{0 / 2}$ \\
\hline 1519.813 & 1000 & 65797.6 & -.005 & $b^{2} G_{9 / 2}-z^{2} H_{11 / 2}^{0}$ & 1384.883 & 80 & 72208.3 & .000 & $b^{4} P_{1 / 2}^{9 / 2}-z^{2} P_{1 / 2}^{9 / 2}$ \\
\hline 1518.518 & 50 & 65853.7 & -.003 & $b^{4} P_{3 / 2}-z^{4} P_{3 / 2}^{0}$ & & 100 & 72307.0 & .000 & $b^{2} F_{5 / 2}^{1 / 2}-y^{2} F_{7 / 2}^{0}$ \\
\hline 1518.257 & 100 & 65865.0 & -.005 & $c^{2} D_{3 / 2}-z^{2} P_{1 / 2}^{0}$ & $\begin{array}{l}1382.992 \\
1381.505\end{array}$ & 200 & 72384.8 & -.002 & $a^{2} F_{5 / 2}^{5 / 2}-z^{4} F_{7 / 2}^{0}$ \\
\hline 1516.497 & 50 & 65941.4 & -.001 & $b^{4} P_{3 / 2}-y^{2} D_{5 / 2}^{0}$ & 1379.578 & $600 \mathrm{H}$ & 72485.9 & -.009 & $b^{4} F_{7 / 2}-y^{4} D_{7 / 2}^{0}$ \\
\hline 1515.932 & 1000 & 65966.0 & -.003 & $b^{4} F_{9 / 2}-z^{4} G_{11 / 2}^{0}$ & 1379.074 & 50 & 72512.4 & -.003 & $a^{2} F_{5 / 2}-y^{2} D_{3 / 2}^{0}$ \\
\hline 1515.595 & 25 & 65980.7 & .000 & $b^{2} F_{5 / 2}-z^{4} P_{5 / 2}^{0}$ & 1375.888 & 50 & 72680.3 & .000 & $b^{2} D_{3 / 2}-z^{2} P_{3 / 2}^{0}$ \\
\hline 1515.162 & 500 & 65999.5 & -.004 & $b^{4} F_{3 / 2}^{3}-z^{4} D_{5 / 2}^{0}$ & 1373.174 & 80 & 72824.0 & -.003 & $b^{2} D_{5 / 2}-z^{4} D_{7 / 2}^{0}$ \\
\hline 1512.556 & 50 & 66113.3 & -.002 & $a^{2} F_{7 / 2}-z^{4} F_{5 / 2}^{0}$ & 1372.007 & $200 \mathrm{D}$ & 72885.9 & .002 & $b^{4} F_{9 / 2}-z^{4} D_{7 / 2}^{0}$ \\
\hline 1509.806 & 500 & 66233.7 & .000 & $b^{4} F_{7 / 2}-z^{4} F_{9 / 2}^{0}$ & 1370.741 & 80 & 72953.2 & .006 & $b^{4} P_{5 / 2}-x^{2} D_{3 / 2}^{0}$ \\
\hline $\begin{array}{l}1509.416 \\
1501.780\end{array}$ & $\begin{array}{l}50 \\
30\end{array}$ & $\begin{array}{l}66250.8 \\
66587.6\end{array}$ & $\begin{array}{l}.000 \\
.000\end{array}$ & $\begin{array}{l}b^{2} F_{7 / 2}-z^{2} D_{s / 2}^{0} \\
a^{2} F^{2}\end{array}$ & 1370.012 & 200 & 72992.1 & --.011 & $b^{4} P_{5 / 2}-z^{2} D_{5 / 2}^{0}$ \\
\hline
\end{tabular}

Table I. Continued (A) 
Table I. Continued

\begin{tabular}{|c|c|c|c|c|c|c|c|c|c|}
\hline $\begin{array}{l}\text { Wavelength } \\
\text { (A) }\end{array}$ & Int. & $\begin{array}{l}\text { Wavenumber } \\
\left(\mathrm{cm}^{-1}\right)\end{array}$ & $\begin{array}{l}\mathrm{O}-\mathrm{C} \\
(\AA)\end{array}$ & Classification & $\begin{array}{l}\text { Wavelength } \\
\text { (A) }\end{array}$ & Int. & $\begin{array}{l}\text { Wavenumber } \\
\left(\mathrm{cm}^{-1}\right)\end{array}$ & $\begin{array}{l}O-C \\
(\AA)\end{array}$ & Classification \\
\hline 1364.336 & $100 \mathrm{~B}$ & 73295.7 & .003 & $a^{2} P_{3 / 2}-z^{4} D_{1 / 2}^{0}$ & 1276.453 & $200 \mathrm{~B}$ & 78342.1 & .003 & $a^{2} F_{7 / 2}-z^{2} F_{s / 2}^{0}$ \\
\hline 1356.792 & 1 & 73703.3 & -.004 & $c^{2} D_{5 / 2}-y^{4} F_{3 / 2}^{0}$ & 1275.561 & 0 & 78396.9 & .004 & $b^{4} P_{3 / 2}-y^{2} P_{3 / 2}^{0}$ \\
\hline 1356.299 & 30 & 73730.1 & -.002 & $b^{2} P_{1 / 2}-x^{2} P_{1 / 2}^{0}$ & 1274.873 & 0 & 78439.2 & .002 & $b^{4} F_{5 / 2}-z^{4} P_{3 / 2}^{0}$ \\
\hline 1354.484 & 400 & 73828.9 & -.005 & $b^{4} F_{5 / 2}^{1 / 2}-y^{4} D_{3 / 2}^{0}$ & 1273.447 & 50 & 78527.0 & .003 & $b^{4} F_{s / 2}-y^{2} D_{5 / 2}^{0}$ \\
\hline 1353.545 & $500 \mathrm{D}$ & 73880.1 & -.003 & $b^{2} F_{5 / 2}-x^{2} D_{s / 2}^{0}$ & 1273.151 & 100 & 78545.3 & .001 & $a^{4} F_{7 / 2}^{3 / 4}-z^{4} G_{5 / 2}^{0}$ \\
\hline 1351.643 & 100 & 73984.0 & -.007 & $b^{2} D_{3 / 2}-z^{2} P_{1 / 2}^{0}$ & 1269.286 & 20 & 78784.5 & .004 & $a^{2} P_{3 / 2}-z^{4} D_{3 / 2}^{0}$ \\
\hline 1351.383 & 3 & 73998.3 & .000 & $b^{4} P_{3 / 2}^{3 / 2}-y^{2} P_{1 / 2}^{0}$ & 1269.155 & 100 & 78792.6 & .003 & $a^{2} F_{5 / 2}-z^{2} G_{7 / 2}^{0}$ \\
\hline 1350.219 & 40 & 74062.1 & -.001 & $b^{4} F_{3 / 2}-z^{2} F_{5 / 2}^{0}$ & $\begin{array}{l}1268.765 \\
1261605\end{array}$ & $\begin{array}{r}100 \\
5\end{array}$ & $\begin{array}{l}78816.8 \\
79264\end{array}$ & $\begin{array}{r}-.001 \\
.004\end{array}$ & $a^{2} F_{5 / 2}-z^{2} F_{5 / 2}^{0}$ \\
\hline 1347.407 & 50 & 74216.6 & -.006 & $c^{2} D_{3 / 2}-y^{2} P_{3 / 2}^{0}$ & 1259.485 & $\begin{array}{l}3 \\
0\end{array}$ & $\begin{array}{l}19264.1 \\
79397.5\end{array}$ & .003 & $\begin{array}{l}a^{4} G_{7 / 2}-2 F_{5 / 2} \\
b^{4} P_{3 / 2}-x^{2} F_{5 / 2}^{0}\end{array}$ \\
\hline 1346.819 & 80 & 74249.0 & .000 & $b^{2} F_{7 / 2}-x^{2} F_{s / 2}^{0}$ & & & & & \\
\hline 1343.080 & 50 & 74455.7 & -.004 & $a^{2} F_{7 / 2}-z^{2} F_{7 / 2}^{0}$ & 1258.253 & $100 \mathrm{~B}$ & 79475.3 & .002 & $b^{2} D_{3 / 2}-x^{2} D_{5 / 2}^{0}$ \\
\hline 1341.000 & 50 & 74571.2 & -.001 & $b^{2} F_{5 / 2}-x^{2} F_{7 / 2}^{0}$ & 1255.924 & ${ }_{0 \mathrm{H}}$ & 79622.7 & .006 & $b^{4} F_{9 / 2}-z^{2} H_{11 / 2}^{0}$ \\
\hline 1340.638 & 200 & 74591.4 & -.002 & $b^{4} F_{7 / 2}-y^{2} D_{5 / 2}^{0}$ & 1255.144 & 300 & 79672.1 & .004 & $a^{4} P_{3 / 2}-z^{4} F_{3 / 2}^{0}$ \\
\hline 1338.695 & 20 & 74699.6 & -.004 & $a^{2} P_{3 / 2}-z^{2} D_{3 / 2}^{0}$ & 1251.095 & 200 & 79930.0 & .005 & $b^{2} D_{5 / 2}-x^{2} D_{5 / 2}^{0}$ \\
\hline 1337.765 & 10 & 74751.5 & -.005 & $b^{2} P_{1 / 2}-x^{4} D_{1 / 2}^{0}$ & 1250.867 & $50 \mathrm{~B}$ & 79944.6 & .002 & $a^{2} D_{3 / 2}-z^{4} D_{1 / 2}^{0}$ \\
\hline 1337.104 & 20 & 74788.5 & .001 & $b^{4} F_{5 / 2}-z^{4} S_{3 / 2}^{0}$ & 1250.176 & 5 & 79988.7 & & $b^{4} P_{s / 2}-y^{2} P_{3 / 2}^{0}$ \\
\hline 1336.823 & 20 & 74804.2 & .003 & $a^{2} F_{3 / 2}-y^{4} D_{5 / 2}^{0}$ & 1249.236 & 1 & 80048.9 & .006 & $b^{4} F_{7 / 2}-z^{2} D_{5 / 2}^{0}$ \\
\hline 1334.574 & 200 & 74930.3 & -.005 & $a^{2} F_{5 / 2}-z^{2} F_{7 / 2}^{0}$ & $\begin{array}{l}1249.049 \\
1248.464\end{array}$ & $\begin{array}{l}50 \\
50 \mathrm{~B}\end{array}$ & $\begin{array}{l}80060.9 \\
80098.4\end{array}$ & $\begin{array}{l}.002 \\
.008\end{array}$ & $\begin{array}{l}d^{2} D_{s / 2}-v^{2} P_{3 / 2}^{0} \\
a^{2} P^{4}-z^{4} D^{0}\end{array}$ \\
\hline 1334.532 & 300 & 74932.6 & -.007 & $a^{2} G_{9 / 2}-z^{4} G_{9 / 2}^{0}$ & $\begin{array}{l}1248.464 \\
1248.426\end{array}$ & $\begin{array}{l}50 \mathrm{~B} \\
50 \mathrm{~B}\end{array}$ & $\begin{array}{l}80098.4 \\
80100.9\end{array}$ & $\begin{array}{l}.008 \\
.006\end{array}$ & $\begin{array}{l}a \\
a^{2} P_{1 / 2}-z^{2} D_{1 / 2}^{1 / 2}-z^{2} F_{0}^{0}\end{array}$ \\
\hline 1332.100 & 300 & 75069.4 & -.002 & $a^{4} P_{3 / 2}-z^{4} G_{5 / 2}^{0}$ & 1240.420 & & & & \\
\hline 1330.005 & 100 & 75187.7 & .003 & $d^{2} D_{5 / 2}-v^{2} D_{5 / 2}^{0}$ & 1248.154 & 10 & 80118.3 & -.003 & $a^{2} S_{1 / 2}-w^{2} P_{3 / 2}^{0}$ \\
\hline 1329.499 & 200 & 75216.3 & .009 & $c^{2} D_{3 / 2}-x^{2} F_{5 / 2}^{0}$ & 1247.374 & 50 & 80168.4 & .006 & $b^{4} F_{3 / 2}-z^{4} P_{1 / 2}^{0}$ \\
\hline 1328.391 & 200 & 75279.0 & -.003 & $a^{2} F_{5 / 2}-y^{4} D_{5 / 2}^{0}$ & 1247.308 & $100 \mathrm{~B}$ & 80172.7 & .001 & $a^{2} D_{3 / 2}-z^{4} D_{5 / 2}^{0}$ \\
\hline 1328.333 & 50 & 75282.3 & -.004 & $a^{2} P_{3 / 2}-y^{2} D_{3 / 2}^{0}$ & 1246.519 & 20 & 80223.4 & .008 & $b^{4} F_{5 / 2}-z^{4} P_{5 / 2}^{0}$ \\
\hline 1328.031 & 50 & 75299.4 & .002 & $b^{2} D_{3 / 2}-x^{2} D_{3 / 2}^{0}$ & 1242.856 & 200 & 80459.8 & .000 & $a^{2} F_{7 / 2}-y^{2} G_{7 / 2}^{0}$ \\
\hline 1327.359 & 50 & 75337.6 & -.001 & $b^{2} D_{3 / 2}-z^{2} D_{5 / 2}^{0}$ & 1241.314 & 20 & 80559.8 & -.001 & $b^{4} P_{1 / 2}-y^{2} P_{3 / 2}^{0}$ \\
\hline 1323.847 & 20 & 75537.4 & -.001 & $b^{4} P_{3 / 2}-x^{2} D_{5 / 2}^{0}$ & 1240.360 & 300 & 80621.8 & -.002 & $b^{2} D_{5 / 2}-x^{2} F_{7 / 2}^{0}$ \\
\hline 1323.515 & 10 & 75556.4 & .002 & $b^{4} P_{5 / 2}-y^{2} F_{7 / 2}^{0}$ & $\begin{array}{l}1239.711 \\
1239408\end{array}$ & 50 & 80664.0 & .005 & $b^{4} F_{7 / 2}-y^{2} G_{9 / 2}^{0}$ \\
\hline 1323.370 & 50 & 75564.7 & .001 & $b^{4} F_{5 / 2}-y^{2} F_{s / 2}^{0}$ & 1239.408 & 50 & 80683.7 & .002 & $b^{4} F_{9 / 2}-x^{2} F_{7 / 2}^{0}$ \\
\hline 1321.772 & 50 & 75656.0 & -.003 & $a^{4} P_{5 / 2}-z^{4} G_{7 / 2}^{0}$ & & 50 & 80840.6 & .001 & $b^{4} F_{3 / 2}-z^{2} P_{3 / 2}^{0}$ \\
\hline 1320.053 & 100 & 75754.5 & .000 & $b^{2} D_{5 / 2}-x^{2} D_{3 / 2}^{0}$ & 1236.271 & 10 & 80888.4 & -.001 & $b^{2} P_{3 / 2}-y^{4} P_{3 / 2}^{0}$ \\
\hline 1319.398 & $100 \mathrm{~B}$ & 75792.1 & .005 & $b^{2} D_{s / 2}-z^{2} D_{s / 2}^{0}$ & 1235.568 & 300 & 80934.4 & -.002 & $a^{2} F_{s / 2}-y^{2} G_{7 / 2}^{0}$ \\
\hline 1317.081 & 100 & 75925.5 & -.001 & $a^{2} D_{5 / 2}-z^{4} D_{5 / 2}^{0}$ & 1235.226 & 100 & 80956.8 & .006 & $b^{2} F_{7 / 2}-y^{4} F_{3 / 2}^{0}$ \\
\hline 1315.679 & 20 & 76006.4 & -.002 & $a^{2} D_{3 / 2}-z^{4} F_{5 / 2}^{0}$ & 1234.805 & 50 & 80984.4 & .004 & $a^{2} F_{5 / 2}-y^{4} D_{3 / 2}^{0}$ \\
\hline 1315.534 & 50 & 76014.8 & .003 & $a^{2} F_{5 / 2}-z^{4} D_{3 / 2}^{0}$ & 1234.725 & 100 & 80989.7 & .000 & $b^{4} P_{s / 2}-x^{2} F_{s / 2}^{0}$ \\
\hline 1313.929 & $50 \mathrm{D}$ & 76107.6 & .000 & $a^{2} P_{3 / 2}-z^{2} S_{1 / 2}^{0}$ & 1232.023 & 200 & 81167.3 & .001 & $a^{2} G_{9 / 2}-z^{4} F_{9 / 2}^{0}$ \\
\hline 1313.014 & 20 & 76160.6 & .003 & $b^{4} P_{1 / 2}-y^{2} P_{1 / 2}^{0}$ & 1231.740 & 50 & 81186.0 & .003 & $a^{2} D_{5 / 2}-z^{4} D_{3 / 2}^{0}$ \\
\hline 1312.282 & $100 \mathrm{H}$ & 76203.1 & .002 & $d^{2} D_{3 / 2}-v^{2} F_{s / 2}^{0}$ & $\begin{array}{l}1229.279 \\
1227.029\end{array}$ & $\begin{array}{r}100 \\
20\end{array}$ & $\begin{array}{l}81348.5 \\
81497.7\end{array}$ & -.005 & $\begin{array}{l}a^{2} D_{3 / 2}-z^{2} D_{3 / 2}^{0} \\
c^{2} D_{3 / 2}-x^{2} P^{0}\end{array}$ \\
\hline 1311.883 & $20 \mathrm{~B}$ & 76226.3 & .000 & $a^{2} G_{9 / 2}-z^{4} F_{7 / 2}^{0}$ & & & & $\begin{array}{r}.001 \\
-.008\end{array}$ & $a^{4} P_{5 / 2}-z^{4} D_{5 / 2}^{0}$ \\
\hline 1311.824 & 50 & 76229.7 & .003 & $b^{4} F_{3 / 2}-y^{4} D_{3 / 2}^{0}$ & 1226.958 & 200 & 81502.4 & .001 & $a^{2} P_{1 / 2}^{5 / 2}-z^{2} D_{3 / 2}^{5 / 2}$ \\
\hline 1310.825 & 10 & 76287.8 & .003 & $b^{4} F_{7 / 2}-z^{4} P_{5 / 2}^{0}$ & & & & & ${ }^{2} P$ \\
\hline 1309.520 & $50 \mathrm{D}$ & 76363.9 & .000 & $d^{2} D_{3 / 2}-v^{2} D_{3 / 2}^{0}$ & 1225.690 & $300 \mathrm{H}$ & 81586.7 & -.002 & $-z^{2} F_{s / 2}^{0}$ \\
\hline 1309.020 & 10 & 76393.0 & .003 & $b^{4} F_{7 / 2}-z^{2} G_{9 / 2}^{0}$ & 1225.563 & 100 & 81595.2 & .003 & $d^{2} D_{3 / 2}-v^{2} P_{1 / 2}^{0}$ \\
\hline 1308.537 & 10 & 76421.2 & .003 & $b^{4} F_{5 / 2}-y^{4} D_{7 / 2}^{0}$ & 1223.208 & 20 & 81752.2 & .004 & $a^{4} F_{5 / 2}-z^{4} G_{5 / 2}^{0}$ \\
\hline 1307.713 & 50 & 76469.4 & .004 & $b^{4} F_{9 / 2}-y^{2} G_{9 / 2}^{0}$ & 1221.894 & $100 \mathrm{~B}$ & 81840.2 & .007 & $a^{2} F_{7 / 2}-z^{2} H_{9 / 2}^{0}$ \\
\hline 1305.806 & 100 & 76581.1 & .002 & $d^{2} D_{5 / 2}-v^{2} F_{7 / 2}^{0}$ & 1220.628 & 10 & 81925.0 & .000 & $c^{2} D_{3 / 2}-y^{4} F_{3 / 2}^{0}$ \\
\hline 1304.783 & 200 & 76641.1 & -.004 & $a^{2} H_{9 / 2}-z^{4} G_{7 / 2}^{0}$ & 1220.547 & 50 & 81930.5 & .006 & $a^{2} D_{3 / 2}-y^{2} D_{3 / 2}^{0}$ \\
\hline 1303.107 & $100 \mathrm{~B}$ & 76739.7 & .000 & $b^{2} F_{5 / 2}-y^{2} P_{3 / 2}^{0}$ & 1220.342 & 50 & 81944.2 & $\begin{array}{l}.004 \\
.007\end{array}$ & $\begin{array}{l}b^{2} G_{7 / 2}-x^{4} D_{3 / 2}^{0} \\
a^{2} F_{5 / 2}-z^{4} S_{3}^{0}\end{array}$ \\
\hline 1301.992 & 200 & 76805.4 & -.003 & $a^{2} H_{11 / 2}-z^{4} G_{9 / 2}^{0}$ & 1218.242 & 100 & 82085.5 & -.005 & $a^{2} P_{1 / 2}^{3 / 2}-y^{2} D_{3 / 2}^{0}$ \\
\hline 1301.214 & 300 & 76851.3 & -.001 & $a^{2} F_{7 / 2}-z^{4} F_{9 / 2}^{0}$ & 1215.857 & $100 \mathrm{H}$ & 82246.5 & -.003 & $a^{2} F_{7 / 2}^{1 / 2}-y^{2} F_{s / 2}^{0 / 2}$ \\
\hline $\begin{array}{l}1297.342 \\
1297.003\end{array}$ & $\begin{array}{r}50 \\
100\end{array}$ & $\begin{array}{l}77080.7 \\
77100.8\end{array}$ & $\begin{array}{l}.000 \\
.000\end{array}$ & $\begin{array}{l}b^{4} F_{7 / 2}-z^{4} D_{7 / 2}^{0} \\
a^{2} D^{0}-z^{2} D_{3}^{0}\end{array}$ & 1214.545 & 100 & 82335.4 & -.003 & $b^{2} D_{3 / 2}^{7 / 2}-y^{2} P_{3 / 2}^{0 / 2}$ \\
\hline 1295.507 & $10 \mathrm{~B}$ & 77189.9 & .001 & $b^{4} F_{3 / 2}^{5 / 2}-z^{4} S_{3 / 2}^{0}$ & 1213.021 & 20 & 82438.8 & .004 & $b^{4} P_{3 / 2}-y^{4} F_{3 / 2}^{0}$ \\
\hline 1293.068 & $200 \mathrm{BD}$ & 77335.5 & -.001 & $a^{4} P_{5 / 2}-z^{4} F_{5 / 2}^{0}$ & 1211.018 & 50 & 82575.2 & .002 & $b^{2} P_{1 / 2}-x^{2} P_{3 / 2}^{0}$ \\
\hline 1292.486 & 20 & 77370.3 & -.006 & $c^{2} D_{s / 2}-y^{4} F_{s / 2}^{0}$ & 1210.290 & 10 & 82624.8 & .007 & $b^{4} F_{3 / 2}-z^{4} P_{5 / 2}^{0}$ \\
\hline 1289.946 & 3 & 77522.6 & -.002 & $b^{2} P_{3 / 2}-x^{2} P_{3 / 2}^{0}$ & 1210.154 & 100 & 82634.1 & .004 & $a^{2} G_{9 / 2}-z^{2} G_{7 / 2}^{0}$ \\
\hline 1289.393 & 100 & 77555.9 & .002 & $a^{2} D_{s / 2}^{5 / 2}-z^{4} F_{7 / 2}^{0}$ & 1209.519 & 100 & 82677.5 & -.003 & $a^{4} P_{5 / 2}-z^{2} D_{3 / 2}^{0}$ \\
\hline 1288.910 & 100 & 77584.9 & -.003 & $a^{2} G_{7 / 2}-z^{4} G_{7 / 2}^{0}$ & 1208.885 & 200 & 82720.9 & .000 & $a^{2} F_{s / 2}-y^{2} F_{s / 2}^{0}$ \\
\hline 1287.274 & 200 & 77683.5 & .000 & $a^{2} D_{5 / 2}^{7 / 2}-y^{2} D_{3 / 2}^{0 / 2}$ & 1207.871 & 200 & 82790.3 & -.003 & $b^{2} D_{5 / 2}-y^{2} P_{3 / 2}^{0}$ \\
\hline 1286.336 & 5 & 77740.2 & .000 & $b^{2} F_{5 / 2}^{5 / 2}-x^{2} F_{5 / 2}^{0 / 2}$ & 1207.386 & 100 & 82823.6 & -.002 & $a^{2} H_{9 / 2}-z^{4} G_{9 / 2}^{0}$ \\
\hline 1285.003 & 20 & 77820.8 & -.003 & $b^{4} P_{5 / 2}^{5 / 2}-x^{2} F_{7 / 2}^{6 / 2}$ & 1206.120 & 100 & 82910.5 & .002 & $a^{2} P_{1 / 2}-z^{2} S_{1 / 2}^{0}$ \\
\hline 1283.103 & 100 & 77936.1 & .003 & $b^{2} D_{3 / 2}-y^{2} P_{1 / 2}^{0}$ & 1204.235 & 100 & 83040.3 & .001 & $a^{2} H_{11 / 2}-z^{4} F_{9 / 2}^{0}$ \\
\hline 1282.612 & 1 & 77965.9 & .003 & $b^{4} F_{3 / 2}-y^{2} F_{5 / 2}^{0}$ & 1203.325 & 50 & 83103.1 & .000 & $a^{2} F_{7 / 2}-y^{4} D_{7 / 2}^{0}$ \\
\hline 1281.252 & 5 & 78048.7 & .001 & $a^{2} P_{3 / 2}-y^{4} D_{5 / 2}^{0}$ & 1202.898 & 300 & 83132.6 & -.001 & $a^{4} P_{5 / 2}-z^{4} F_{7 / 2}^{0}$ \\
\hline 1280.079 & 200 & 78120.2 & -.001 & $a^{4} P_{1 / 2}-z^{4} F_{3 / 2}^{0}$ & 1201.058 & 50 & 83259.9 & .002 & $a^{4} P_{\mathrm{s} / 2}-y^{2} D_{3 / 2}^{0}$ \\
\hline 1277.819 & 50 & 78258.3 & -.002 & $c^{2} D_{3 / 2}-y^{4} F_{3 / 2}^{0}$ & 1199.961 & 200 & 83336.0 & -.005 & $b^{2} D_{3 / 2}-x^{2} F_{5 / 2}^{0}$ \\
\hline 1276.842 & $100 \mathrm{~B}$ & 78318.2 & .001 & $a^{2} F_{9 / 2}-z^{2} G_{7,2}^{0}$ & 1198.598 & 5 & 83430.8 & .000 & $a^{2} G_{7 / 2}-z^{4} D_{5 / 2}^{0}$ \\
\hline
\end{tabular}

Table I. Continued (A) 
Table I. Continued

\begin{tabular}{|c|c|c|c|c|c|c|c|c|c|}
\hline $\begin{array}{l}\text { Wavelength } \\
(\AA)\end{array}$ & Int. & $\begin{array}{l}\text { Wavenumber } \\
\left(\mathrm{cm}^{-1}\right)\end{array}$ & $\begin{array}{l}O-C \\
(\AA)\end{array}$ & Classification & $\begin{array}{l}\text { Wavelength } \\
(\AA)\end{array}$ & Int. & $\begin{array}{l}\text { Wavenumber } \\
\left(\mathrm{cm}^{-1}\right)\end{array}$ & $\begin{array}{l}O-C \\
(\AA)\end{array}$ & Classification \\
\hline 1196.495 & 50 & 83577.4 & .001 & $a^{2} F_{5 / 2}-y^{4} D_{7 / 2}^{0}$ & 1122.859 & 30 & 89058.4 & .002 & $a^{2} H_{9 / 2}-z^{4} F_{9 / 2}^{0}$ \\
\hline 1196.321 & 200 & 83589.6 & -.004 & $a^{4} F_{9 / 2}^{5 / 2}-z^{4} G_{7 / 2}^{0 / 2}$ & 1121.633 & $10 \mathrm{H}$ & 89155.7 & -.002 & $b^{2} G_{9 / 2}^{9 / 2}-x^{4} D_{7 / 2}^{9 / 2}$ \\
\hline 1194.325 & 50 & 83729.3 & .002 & $b^{4} F_{3 / 2}-z^{2} P_{3 / 2}^{0}$ & 1119.710 & 500 & 89308.8 & -.008 & $a^{4} F_{5,2}-z^{4} G_{7 / 2}^{0}$ \\
\hline 1194.190 & 50 & 83738.8 &.- .004 & $b^{2} G_{9 / 2}^{3 / 2}-y^{4} F_{7 / 2}^{0,2}$ & 1117.286 & 20 & 89502.6 & -.001 & $a^{4} P_{1 / 2}-z^{2} S_{1 / 2}^{0}$ \\
\hline 1193.788 & 3 & 83767.0 & .005 & $a^{2} G_{7 / 2}-z^{4} G_{9 / 2}^{0 / 2}$ & 1116.813 & 5 & 89540.5 & .000 & $a^{4} P_{5 / 2}-z^{2} G_{7 / 2}^{0}$ \\
\hline 1193.447 & 200 & 83790.9 & -.004 & $b^{2} D_{5 / 2}-x^{2} F_{5 / 2}^{0}$ & 1116.512 & 1 & 89564.6 & -.002 & $a^{4} P_{5 / 2}-z^{2} F_{5 / 2}^{0}$ \\
\hline 1191.870 & 50 & 83901.8 & -.004 & $\begin{array}{l}D-D_{5 / 2}-x^{4} F_{5 / 2} \\
c^{2} D_{5 / 2}-x^{4} D_{5 / 2}^{0}\end{array}$ & 1115.863 & 100 & 89616.7 & -.001 & $b^{2} D_{3 / 2}-x^{2} P_{1 / 2}^{0}$ \\
\hline 1191.231 & 10 & 83946.8 & .008 & $\begin{array}{l}c^{2} D_{5 / 2}-x^{4} D_{5 / 2}^{5} \\
b^{4} F_{5 / 2}-x^{2} D_{3 / 2}^{0}\end{array}$ & 1115.491 & 50 & 89646.6 & -.001 & $a^{4} P_{3 / 2}-z^{2} D_{3 / 2}^{0}$ \\
\hline 1190.982 & $200 \mathrm{H}$ & 83964.3 & -.004 & & 1113.934 & 500 & 89771.9 & -.001 & $a^{4} F_{9 / 2}-z^{4} G_{9 / 2}^{0}$ \\
\hline 1190.643 & 200 & 83988.2 & -.003 & $\begin{array}{l}a^{2} D_{5 / 2}-z^{2} G_{7 / 2}^{0} \\
a^{2} D_{5 / 2}-z^{2} F_{5 / 2}^{0}\end{array}$ & 1113.704 & 10 & 89790.5 & -.004 & $c^{2} D_{5 / 2}-x^{4} D_{7 / 2}^{0}$ \\
\hline 1188.813 & 100 & 84117.5 & .000 & $a^{2} H_{9 / 2}-z^{4} F_{7 / 2}^{0}$ & 1112.430 & 500 & 89893.3 & -.003 & $a^{4} F_{3 / 2}-z^{4} F_{3 / 2}^{0}$ \\
\hline 1187.831 & 0 & 84187.1 & .002 & $b^{4} F_{7 / 2}-x^{2} D_{5 / 2}^{7 / 2}$ & 1111.081 & 30 & 90002.4 & .000 & $a^{2} G_{7 / 2}-z^{4} F_{9 / 2}^{0}$ \\
\hline 1186.169 & $300 \mathrm{H}$ & 84305.0 & -.006 & $a^{4} P_{3 / 2}-z^{4} F_{5 / 2}^{0}$ & 1109.263 & 20 & 90149.9 & -.001 & $a^{2} P_{3 / 2}-z^{4} P_{5 / 2}^{0}$ \\
\hline 1185.214 & 3 & 84372.9 & .001 & $c^{2} D_{5 / 2}^{3 / 2}-y^{4} F_{7 / 2}^{0}$ & 1108.284 & 500 & 90229.6 & -.004 & $a^{4} P_{3 / 2}-y^{2} D_{3 / 2}^{0}$ \\
\hline 1180.671 & 300 & 84697.6 & -.001 & $a^{2} D_{3 / 2}^{5 / 2}-y^{4} D_{s / 2}^{0}$ & 1107.341 & 50 & 90306.4 & -.002 & $b^{4} P_{1 / 2}-x^{4} D_{3 / 2}^{0}$ \\
\hline 1180.437 & 3 & 84714.4 & .002 & $a^{2} P_{3 / 2}-z^{4} S_{3 / 2}^{0}$ & 1106.990 & 1 & 90335.1 & -.005 & $c^{2} D_{5 / 2}-w^{2} D_{3 / 2}^{0}$ \\
\hline 1179.579 & 200 & 84776.0 & -.001 & $\begin{array}{l}a \\
a^{2} G_{3 / 2}-y^{2} S_{3 / 2} G_{0}^{0}\end{array}$ & 1106.892 & 2 & 90343.1 & .000 & $c^{2} D_{3 / 2}-x^{2} P_{3 / 2}^{0}$ \\
\hline 1178.163 & 5 & 84877.9 & .008 & & 1106.149 & 100 & 90403.7 & -.005 & $a^{2} D_{3 / 2}-y^{4} D_{3 / 2}^{0}$ \\
\hline 1175.620 & $100 \mathrm{D}$ & 85061.5 &.- .001 & $\begin{array}{l}b^{4} F_{7 / 2}-x^{2} F_{7 / 2}^{0} \\
a^{2} G_{7,3}-z^{4} F_{7,0}^{0}\end{array}$ & 1104.997 & 0 & 90498.0 & .011 & $b^{2} D_{5 / 2}-y^{4} F_{5 / 2}^{0}$ \\
\hline 1175.166 & 200 & 85094.4 & -.001 & $\begin{array}{l}a^{2} G_{7 / 2}-z F_{7 / 2}^{0} \\
a^{2} G_{9 / 2}-z^{4} G_{11 / 2}^{0}\end{array}$ & 1104.659 & 100 & 90525.7 & -.001 & $a^{2} H_{9 / 2}-z^{2} G_{7 / 2}^{0}$ \\
\hline 1174.216 & 50 & 85163.2 & .002 & $a^{2} D_{3 / 2}^{1 / 2}-y^{4} D_{1 / 2}^{0}$ & 1104.269 & $100 \mathrm{H}$ & 90557.6 & -.001 & $a^{2} P_{1 / 2}-y^{4} D_{3 / 2}^{0}$ \\
\hline 1173.590 & 100 & 85208.6 & .003 & $a^{2} F_{7 / 2}-y^{2} D_{5 / 2}^{0}$ & 1103.293 & 20 & 90637.8 & .002 & $b^{2} D_{3 / 2}-x^{4} D_{1 / 2}^{0}$ \\
\hline 1172.469 & 800 & 85290.1 & -.002 & $a^{4} F_{3 / 2}^{7 / 2}-z^{4} G_{5 / 2}^{0}$ & 1102.937 & 300 & 90667.0 & -.001 & $a^{2} F_{y / 2}^{3 / 2}-z^{2} D_{5 / 2}^{0}$ \\
\hline 1172.092 & 5 & 85317.5 & .001 & $a^{2} P_{1 / 2}-y^{4} D_{1 / 2}^{0}$ & 1102.471 & 5 & 90705.3 & -.001 & $b^{2} F_{7 / 2}-w^{2} D_{5 / 2}^{0}$ \\
\hline 1170.501 & 10 & 85433.5 & .000 & $a^{2} D_{3 / 2}-z^{4} D_{3 / 2}^{0}$ & 1101.725 & 50 & 90766.8 & .000 & $a^{2} D_{5 / 2}-z^{4} P_{3 / 2}^{0}$ \\
\hline 1169.718 & 100 & 85490.7 & .000 & $a^{2} P_{3 / 2}-y^{2} F_{5 / 2}^{0}$ & 1100.660 & 300 & 90854.6 & .000 & $a^{2} D_{5 / 2}-y^{2} D_{5 / 2}^{0}$ \\
\hline 1168.391 & 50 & 85587.8 & .000 & $a^{2} P_{1 / 2}^{3 / 2}-z^{4} D_{3 / 2}^{5 / 2}$ & 1099.143 & $5 \mathrm{H}$ & 90980.0 & .000 & $b^{2} F_{5 / 2}-x^{4} D_{5 / 2}^{0}$ \\
\hline 1167.169 & $300 \mathrm{H}$ & 85677.4 & .005 & $a^{4} P_{5 / 2}^{1 / 2}-z^{2} F_{7 / 2}^{0 / 2}$ & 1099.046 & 800 & 90988.0 & -.003 & $a^{4} F_{5 / 2}-z^{4} F_{5 / 2}^{0}$ \\
\hline 1165.668 & 1 & 85787.7 & .003 & $c^{2} D_{5 / 2}^{7 / 2}-x^{2} P_{3 / 2}^{0 / 2}$ & 1098.239 & 200 & 91054.9 & -.001 & $a^{4} P_{3 / 2}-z^{2} S_{1 / 2}^{0}$ \\
\hline 1163.767 & 5 & 85927.9 & .004 & $a^{2} S_{1 / 2}-v^{2} D_{3 / 2}^{0}$ & 1098.110 & 30 & 91065.6 & .005 & $a^{4} F_{9 / 2}-z^{4} F_{7 / 2}^{0}$ \\
\hline 1163.588 & 10 & 85941.1 & .001 & $b^{2} P_{1 / 2}-y^{4} P_{3 / 2}^{0}$ & 1097.651 & 300 & 91103.6 & -.002 & $a^{2} F_{5 / 2}-x^{2} D_{3 / 2}^{0}$ \\
\hline 1162.430 & 200 & 86026.7 & -.001 & $a^{4} P_{s / 2}-y^{4} D_{s / 2}^{0}$ & 1097.203 & 2 & 91140.8 & .007 & $a^{2} F_{5 / 2}-z^{2} D_{5 / 2}^{0}$ \\
\hline 1161.426 & $100 \mathrm{~B}$ & 86101.1 & .000 & $a^{4} F_{7 / 2}-z^{4} G_{7 / 2}^{0}$ & 1095.844 & 100 & 91253.9 & .002 & $a^{2} P_{3,2}-z^{2} P_{3 / 2}^{0}$ \\
\hline \multirow{2}{*}{1161.362} & \multirow{2}{*}{500} & \multirow{2}{*}{86105.8} & $(-.003$ & $a^{2} D_{5 / 2}-y^{2} G_{7 / 2}^{0}$ & 1095.511 & 50 & 91281.6 & .004 & $a^{2} F_{7 / 2}-y^{2} G_{9 / 2}^{0}$ \\
\hline & & & .002 & $b^{4} P_{3 / 2}-y^{4} F_{5 / 2}^{0}$ & 1094.970 & 200 & 91326.7 & .002 & $a^{2} G_{9 / 2}^{1 / 2}-z^{2} G_{9 / 2}^{0}$ \\
\hline \multirow[t]{2}{*}{1160.677} & \multirow{2}{*}{10} & \multirow{2}{*}{86156.6} & -.009 & $a^{2} D_{5 / 2}-y^{4} D_{3 / 2}^{0}$ & 1094.527 & 400 & 91363.7 & -.004 & $a^{2} D_{3 / 2}-z^{4} S_{3 / 2}^{0}$ \\
\hline & & & .002 & $a^{2} G_{9 / 2}-z^{2} H_{9 / 2}^{0}$ & 1094.262 & 20 & 91385.8 & .000 & $b^{2} G_{7 / 2}-y^{4} P_{5 / 2}^{0}$ \\
\hline 1158.001 & $20 \mathrm{H}$ & 86355.7 & -.002 & $a^{4} F_{5 / 2}-z^{4} F_{3 / 2}^{0}$ & 1093.477 & 2 & 91451.4 & .001 & $b^{2} F_{5 / 2}-y^{4} F_{7 / 2}^{0}$ \\
\hline 1153.894 & 100 & 86663.1 & -.004 & $\begin{array}{l}a+F_{5 / 2}-z^{2} F_{3 / 2} \\
a^{2} H_{6,2}-z^{2} F^{0}\end{array}$ & 1093.259 & 800 & 91469.6 & -.002 & $a^{2} G_{7 / 2}-z^{2} G_{\eta / 2}^{0}$ \\
\hline 1153.523 & 100 & 86690.9 & -.002 & $\begin{array}{l}a^{2} H_{9 / 2}-z^{2} F_{7 / 2}^{0} \\
a^{4} P_{1 / 2}-z^{4} D_{1 / 2}^{0}\end{array}$ & 1092.973 & 200 & 91493.6 & -.001 & $a^{2} G_{7 / 2}-z^{2} F_{5 / 2}^{0}$ \\
\hline 1152.567 & 100 & 86762.9 & -.003 & $a^{4} P_{3 / 2}^{1 / 2}-z^{4} D_{3 / 2}^{1 / 2}$ & 1092.687 & 100 & 91517.5 & .001 & $a^{2} P_{1 / 2}-z^{4} S_{3 / 2}^{0}$ \\
\hline 1151.652 & 0 & 86831.8 & .003 & $b^{2} D_{5 / 2}-y^{4} F_{3 / 2}^{0}$ & 1090.833 & 3 & 91673.1 & .000 & $c^{2} D_{3 / 2}-w^{2} D_{5 / 2}^{0}$ \\
\hline 1150.674 & 50 & 86905.6 & .000 & $a^{2} F_{7 / 2}-z^{4} P_{\xi / 2}^{0 / 2}$ & 1090.132 & 3 & 91732.0 & .005 & $a^{4} P_{5 / 2}-y^{4} D_{3 / 2}^{0}$ \\
\hline 1149.860 & 50 & 86967.1 & .002 & $a^{2} H_{11 / 2}^{1 / 2}-z^{4} G_{11 / 2}^{0}$ & 1088.026 & $50 \mathrm{~B}$ & 91909.6 & -.001 & $a^{4} P_{1 / 2}-y^{4} D_{1 / 2}^{0}$ \\
\hline 1149.287 & $3 \mathrm{H}$ & 87010.5 & .004 & $a^{2} F_{7 / 2}-z^{2} G_{9 / 2}^{0}$ & 1087.582 & $20 \mathrm{D}$ & 91947.1 & .001 & $a^{4} F_{7 / 2}-z^{4} D_{5 / 2}^{0}$ \\
\hline 1147.892 & 500 & 87116.2 & -.003 & $a^{2} D_{5 / 2}^{7 / 2}-z^{4} S_{3 / 2}^{0 / 2}$ & 1086.786 & 100 & 92014.4 & .000 & $a^{2} G_{9,2}-z^{4} D_{7 / 2}^{0}$ \\
\hline 1143.914 & 50 & 87419.2 & .000 & $a^{2} G_{9 / 2}-y^{4} D_{7 / 2}^{0 / 2}$ & 1084.838 & 50 & 92179.7 & -.001 & $a^{4} P_{1 / 2}-z^{4} D_{3 / 2}^{0}$ \\
\hline 1143.004 & 3 & 87488.8 & .000 & $b^{2} F_{0,2}-x^{4} D^{0}$ & 1083.616 & 300 & 92283.6 & .000 & $a^{4} F_{7 / 2}-z^{4} G_{9 / 2}^{0}$ \\
\hline 1141.462 & 100 & 87606.9 & -.003 & $a^{2} G_{7 / 2}^{7 / 2}-z^{2} F_{5 / 2}^{0}$ & 1080.484 & $40 \mathrm{H}$ & 92551.1 & .003 & $a^{2} D_{5 / 2}-z{ }^{4} P_{5 / 2}^{0}$ \\
\hline 1141.371 & $1 \mathrm{H}$ & 87613.9 & .000 & $d^{2} D_{3 / 2}-v^{2} P_{3 / 2}^{7 / 2}$ & & $100 \mathrm{H}$ & 92557.5 & -.001 & $a^{2} P_{3 / 2}-z^{2} P_{1 / 2}^{0}$ \\
\hline 1140.335 & $10 \mathrm{HB}$ & 87693.5 & -.002 & $a^{2} P_{3 / 2}-z^{4} P_{1 / 2}^{0}$ & 1079.478 & 50 & 92637.4 & .001 & $b^{4} P_{3 / 2}-x^{4} D_{5 / 2}^{0}$ \\
\hline 1140.268 & 500 & 87698.7 & -.005 & $a^{2} F_{7 / 2}-z^{4} D_{7 / 2}^{0}$ & 1079.135 & 2 & 92666.8 & .004 & $a^{2} H_{9 / 2}-y^{2} G_{7 / 2}^{0}$ \\
\hline 1138.422 & 5 & 87840.9 & .004 & $b^{4} P_{1 / 2}-x^{2} P_{1 / 2}^{0}$ & 1078.839 & 60 & 92692.2 & .003 & $a^{4} P_{5 / 2}-z^{4} S_{3 / 2}^{0}$ \\
\hline 1137.758 & 100 & 87892.2 & -.001 & $a^{2} D_{s / 2}-y^{2} F_{s / 2}^{0}$ & 1076.824 & 0 & 92865.7 & .009 & $b^{2} F_{s / 2}-x^{2} P_{3 / 2}^{0}$ \\
\hline 1136.934 & 100 & 87955.9 & -.003 & $a^{2} G_{7 / 2}-y^{4} D_{s / 2}^{0}$ & 1075.439 & 40 & 92985.3 & .002 & $a^{2} H_{9 / 2}-z^{4} G_{11 / 2}^{0}$ \\
\hline 1135.980 & 100 & 88029.7 & .000 & $a^{2} H_{11 / 2}^{1}-z^{2} H_{9 / 2}^{0}$ & 1074.748 & 3 & 93045.1 & .000 & $b^{2} P_{3 / 2}-w^{2} P_{3 / 2}^{0}$ \\
\hline 1135.143 & 500 & 88094.6 & -.005 & $a^{4} P_{1 / 2}-z^{2} D_{3 / 2}^{0}$ & 1072.963 & 800 & 93199.9 & .000 & $a^{2} H_{11 / 2}-z^{2} G_{9 / 2}^{0}$ \\
\hline 1134.778 & 2 & 88123.0 & .002 & $b^{4} F_{5 / 2}-x^{2} D_{5 / 2}^{0}$ & 1072.597 & 50 & 93231.7 & .003 & $a^{2} F_{7 / 2}-y^{2} F_{7 / 2}^{0}$ \\
\hline 1134.138 & 2 & 88172.7 & .001 & $a^{2} F_{5 / 2}-z^{4} D_{7 / 2}^{0}$ & 1071.314 & 30 & 93343.3 & -.006 & $-y^{4} P_{5 / 2}^{0}$ \\
\hline 1133.329 & 100 & 88235.6 & -.003 & $a^{2} D_{3 / 2}-z^{2} F_{s / 2}^{0}$ & & & & & $\begin{array}{l}-z^{4} D_{7 / 2}^{0} \\
-v^{4} F^{0}\end{array}$ \\
\hline 1133.237 & 20 & 88242.8 & .003 & $a^{4} P_{3 / 2}-z^{4} D_{1 / 2}^{0}$ & 1070.989 & 5 & 93371.6 & .004 & $b^{2} G_{9 / 2}-y^{4} F_{9 / 2}^{0}$ \\
\hline 1131.664 & 30 & 88365.5 & -.002 & $a^{2} P_{3 / 2}-z^{4} P_{3 / 2}^{0}$ & 1070.932 & 0 & 93376.6 & .009 & $b^{2} F_{7 / 2}-x^{4} D_{\gamma / 2}^{0}$ \\
\hline 1130.539 & $300 \mathrm{H}$ & 88453.4 &.- .003 & $a^{2} P_{3 / 2}^{0}-y^{2} D_{3 / 2}^{0}$ & 1070.847 & 0 & 93384.0 & .003 & $b^{4} F_{3 / 2}-y^{2} P_{3 / 2}^{0}$ \\
\hline 1130.310 & $100 \mathrm{H}$ & 88471.3 & -.002 & $a^{4} P_{3 / 2}-z^{4} D_{5 / 2}^{0}$ & 1069.959 & 5 & 93461.5 & .002 & $a^{4} P_{3 / 2}-y^{4} D_{1 / 2}^{0}$ \\
\hline 1130.146 & 50 & 88484.1 & .001 & $a^{2} F_{5 / 2}-z^{2} P_{3 / 2}^{0}$ & 1068.628 & 500 & 93577.9 & -.002 & $a^{4} F_{7 / 2}-z^{4} F_{7 / 2}^{0}$ \\
\hline $\begin{array}{l}1127.691 \\
1127.777\end{array}$ & 20 & 88676.8 & .003 & $a^{4} P_{1 / 2}-y^{2} D_{3 / 2}^{0}$ & 1068.247 & 500 & 93611.3 & -.003 & ${ }^{2}-y^{2} G_{7 / 2}^{0}$ \\
\hline 1127.777 & 5 & 88748.7 & .001 & $a^{2} D_{5,3}$ & & & & & \\
\hline
\end{tabular}

Table I. Continued 
Table I. Continued

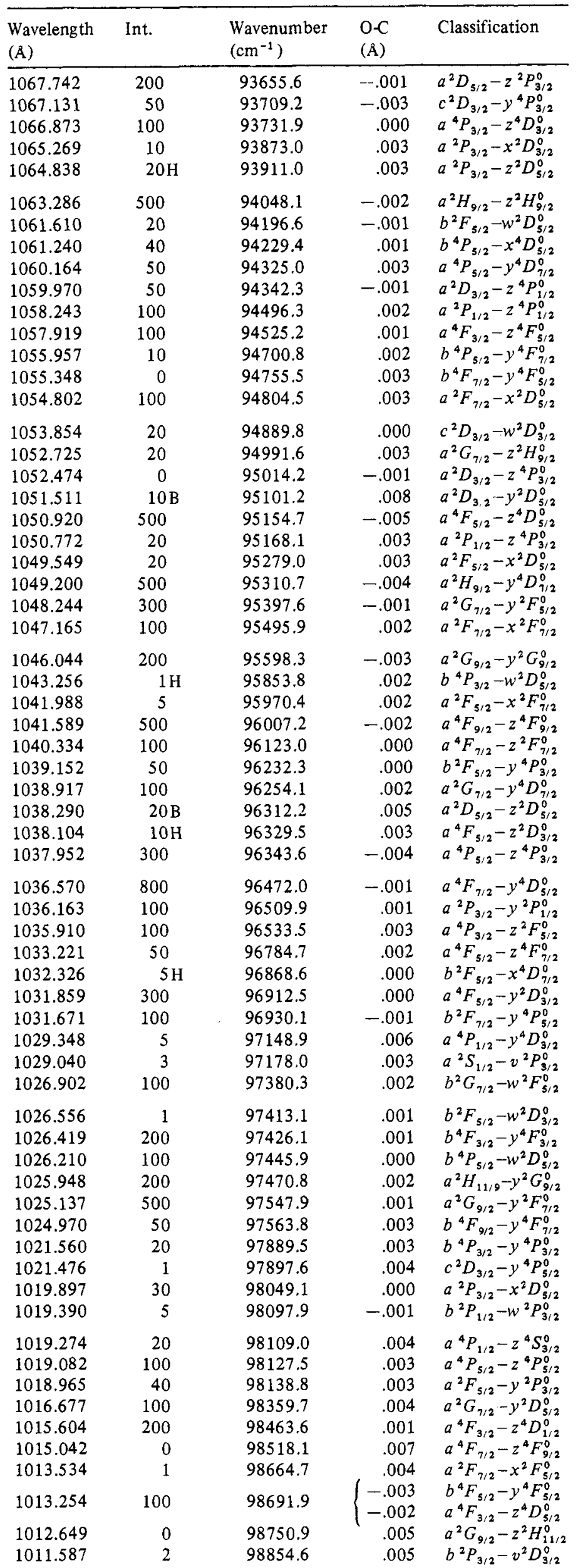

Table I. Continued

Wavelength Int. Wavenumber $O C$ Classification
$(\AA)$

$\left(\mathrm{cm}^{-1}\right)$

$(\AA)$

\begin{tabular}{lllll}
\hline 1011.354 & 30 & 98877.3 & .003 & $a^{2} D_{s / 2}-y^{2} F_{7 / 2}^{0}$
\end{tabular}

$\begin{array}{lllll}1010.957 & 40 \mathrm{~B} & 98916.2 & .007 & b^{2} D_{5 / 2}-x^{2} P_{3 / 2}^{0}\end{array}$

$\begin{array}{lllll}1010.914 & 60 \mathrm{~B} & 98920.4 & .002 & a^{4} P_{5 / 2}-z^{4} D_{7 / 2}^{0}\end{array}$

$\begin{array}{lllll}1009.384 & 5 & 99070.3 & .004 & b^{4} P_{3 / 2}-w^{2} D_{3 / 2}^{0}\end{array}$

$\begin{array}{lllll}1008.681 & 100 & 99139.4 & .002 & a^{2} F_{5 / 2}-x^{2} F_{s / 2}^{0}\end{array}$

$\begin{array}{lllll}1008.006 & 5 & 99205.8 & .004 & a^{2} D_{3 / 2}-z^{2} P_{1 / 2}^{0}\end{array}$

$\begin{array}{lllll}1007.889 & 2 & 99217.3 & .008 & a^{2} H_{9 / 2}-z^{2} G_{9 / 2}^{0}\end{array}$

$\begin{array}{lllll}1007.744 & 5 & 99231.6 & .004 & a^{4} P_{5 / 2}-z^{2} P_{3 / 2}^{0}\end{array}$

$\begin{array}{lllll}1006.669 & 80 & 99337.5 & -.001 & c^{2} D_{s / 2}-w^{2} F_{s / 2}^{0}\end{array}$

$\begin{array}{lllll}1006.439 & 0 & 99360.2 & .002 & a^{2} P_{1 / 2}-z^{2} P_{1 / 2}^{0}\end{array}$

$\begin{array}{lllll}1003.860 & 500 & 99615.5 & .001 & a^{4} F_{9 / 2}-y^{2} G_{7 / 2}^{0}\end{array}$

$\begin{array}{lllll}1003.230 & 5 & 99678.0 & .010 & a^{4} F_{5 / 2}-y^{4} D_{5 / 2}^{0}\end{array}$

$\begin{array}{lllll}1002.753 & 50 & 99725.5 & .004 & b^{4} P_{3 / 2}-y^{4} P_{1 / 2}^{0}\end{array}$

$\begin{array}{lllll}1002.091 & 1 & 99791.3 & .006 & b^{2} D_{3 / 2}-w^{2} D_{s / 2}^{0}\end{array}$

$\begin{array}{lllll}1001.883 & 200 & 99812.1 & .001 & a^{2} G_{9 / 2}-x^{2} F_{9 / 2}^{0}\end{array}$

$\begin{array}{lllll}1001.335 & 100 & 99866.7 & .005 & a^{4} F_{3 / 2}-z^{2} D_{3 / 2}^{0}\end{array}$

$\begin{array}{lllll}1000.953 & 5 & 99904.8 & .008 & a^{2} H_{9,2}-z^{4} D_{7 / 2}^{0}\end{array}$

$\begin{array}{lllll}1000.664 & 200 & 99933.6 & .003 & a^{4} F_{9 / 2}-z^{4} G_{11 / 2}^{0}\end{array}$

$\begin{array}{lllll}1000.142 & 80 & 99985.8 & .000 & a^{4} F_{7 / 2}-z^{2} G_{7 / 2}^{0}\end{array}$

$999.435 \quad 1 \mathrm{H} \quad 100056.5 \quad 003 \quad a^{2} G_{7 / 2}-z^{4} P_{5 / 2}^{0}$

$\begin{array}{lllll}995.869 & 50 & 100414.8 & .002 & a^{4} F_{5 / 2}-z^{4} D_{3 / 2}^{0}\end{array}$

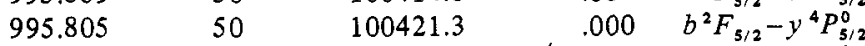

$100450.1 \quad\left(\begin{array}{ll}-.002 & a^{4} F_{3 / 2}-y^{2} D_{3 / 2}^{0}\end{array}\right.$

$a^{2} D_{5 / 2}-x^{2} D_{5 / 2}^{0}$

$\begin{array}{lllll}993.795 & 200 & 100624.4 & .000 & a^{2} H_{1 / 2}-z^{2} H_{1 / 2}^{0}\end{array}$

$\begin{array}{lllll}993.418 & 10 \mathrm{HB} & 100662.6 & .001 & b^{4} P_{5 / 2}-w^{2} D_{3 / 2}^{0}\end{array}$

$\begin{array}{lllll}992.760 & 40 \mathrm{H} & 100729.3 & .001 & b^{4} F_{5 / 2}-x^{4} D_{3 / 2}^{0}\end{array}$

$\begin{array}{lllll}990.995 & 5 & 100908.7 & .002 & a^{2} P_{3 / 2}-y^{2} P_{3 / 2}^{0}\end{array}$

$\begin{array}{lllll}990.745 & 50 & 100934.1 & .000 & b^{2} G_{9 / 2}-w^{2} F_{7 / 2}^{0}\end{array}$

$\begin{array}{lllll}989.187 & 2 & 101093.1 & -.001 & b^{4} G_{3 / 2}-y^{4} F_{s / 2}^{0}\end{array}$

$\begin{array}{lllll}987.819 & 1 \mathrm{H} & 101233.1 & .002 & b^{4} P_{1 / 2}-w^{2} D_{3 / 2}^{0}\end{array}$

$\begin{array}{lllll}987.410 & 30 & 101275.1 & .004 & a^{4} F_{3 / 2}-z \\ & 2 & S_{1 / 2}^{0}\end{array}$

$\begin{array}{lllll}987.293 & 50 & 101287.1 & .002 & b^{4} F_{7 / 2}-x^{4} D_{s / 2}^{0}\end{array}$

$\begin{array}{lllll}987.073 & 1 \mathrm{H} & 101309.6 & .008 & c^{2} D_{5 / 2}-w^{2} P_{3 / 2}^{0}\end{array}$

$\begin{array}{lllll}983.414 & 20 & 101686.6 & .005 & b^{4} F_{3 / 2}-x^{4} D_{1 / 2}^{0}\end{array}$

$\begin{array}{lllll}982.716 & 40 & 101758.8 & .000 & b^{4} F_{7 / 2}-y^{4} F_{7 / 2}^{0}\end{array}$

$\begin{array}{lllll}981.830 & 0 & 101850.6 & .006 & a^{4} P_{5 / 2}-x^{2} D_{3 / 2}^{0}\end{array}$

$981.462 \quad 5 \quad 101888.8 \quad\left\{\begin{aligned}-.004 & b^{4} P_{1 / 2}-y^{4} P_{1 / 2}^{0} \\ .003 & a^{4} P_{5 / 2}-z^{2} D_{5}^{0}\end{aligned}\right.$

$\begin{array}{lllll}979.643 & 0 & 102078.0 & .007 & b^{4} P_{3 / 2}-y^{4} P_{s / 2}^{0}\end{array}$

$\begin{array}{lllll}979.169 & 2 & 102127.4 & -.001 & a^{4} F_{7 / 2}-y^{2} G_{7 / 2}^{0}\end{array}$

$\begin{array}{lllll}977.916 & 1 & 102258.3 & .005 & a^{4} F_{9 / 2}-y^{4} D_{7 / 2}^{0}\end{array}$

$\begin{array}{lllll}971.580 & 0 & 102925.1 & -.004 & b^{2} F_{7 / 2}-w^{2} F_{5 / 2}^{0}\end{array}$

$\begin{array}{lllll}971.048 & 40 & 102981.5 & -.003 & b^{4} F_{9 / 2}-x^{4} D_{7 / 2}^{0}\end{array}$

$\begin{array}{lllll}969.379 & 10 & 103158.8 & .000 & a^{2} D_{3 / 2}-y^{2} P_{1 / 2}^{0}\end{array}$

$\begin{array}{lllll}969.059 & 5 & 103192.9 & .000 & a^{4} F_{5 / 2}-z^{2} G_{7 / 2}^{0}\end{array}$

$968.836 \quad 0 \quad 103216.6 \quad-.001 \quad a^{4} F_{3 / 2}-y^{4} D_{s / 2}^{0}$

\begin{tabular}{lll|ll}
967.961 & 10 & 103309.9 & .002 & $a^{4} F_{5 / 2}-z^{2} F_{5 / 2}^{0}$ \\
9004 & $a^{2} D_{s / 2}-y^{2} P_{3 / 2}^{0}$
\end{tabular}

$\begin{array}{lllll}966.289 & 0 & 103488.7 & .004 & a^{2} H_{9 / 2}-y^{2} G_{9 / 2}^{0}\end{array}$

$\begin{array}{lllll}966.108 & 2 & 103508.1 & .000 & a^{4} F_{7 / 2}-z^{2} H_{9 / 2}^{0}\end{array}$

$962.334 \quad 10 \quad 103914.0 \quad-.002 \quad a^{4} F_{7 / 2}-y^{2} F_{5 / 2}^{0}$

$\begin{array}{lllll}961.976 & 10 & 103952.7 & -.001 & a^{4} F_{3 / 2}-z^{4} D_{3 / 2}^{0}\end{array}$

$\begin{array}{lllll}957.554 & 0 & 104432.8 & .003 & a^{2} G_{7 / 2}-y^{2} G_{9 / 2}^{0}\end{array}$

$956.899 \quad 10 \quad 104504.2 \quad-.005 \quad b^{4} F_{7 / 2}-w^{2} D_{5 / 2}^{0}$

$\begin{array}{lllll}955.124 & 1 & 104698.4 & -.005 & a^{2} D_{3 / 2}-x^{2} D_{5 / 2}^{0}\end{array}$

$\begin{array}{lllll}952.351 & 1 & 105003.3 & -.004 & b^{2} G_{7 / 2}-v^{2} F_{\mathrm{s} / 2}^{0}\end{array}$

$\begin{array}{lllll}951.498 & 0 & 105097.4 & -.003 & a^{4} P_{3 / 2}-z^{4} P_{5 / 2}^{6}\end{array}$

$\begin{array}{lllll}950.352 & 0 & 105224.2 & -.008 & b^{4} F_{5 / 2}^{3 / 2}-x^{4} D_{5 / 2}^{0}\end{array}$

$\begin{array}{lllll}950.275 & 0 & 105232.7 & -.007 & b^{2} P_{3 / 2}-v^{2} D_{5 / 2}^{0}\end{array}$

$\begin{array}{lllll}948.412 & 10 & 105439.4 & -.002 & a^{2} H_{9 / 2}^{3 / 2}-y^{2} F_{7 / 2}^{0}\end{array}$

$\begin{array}{lllll}946.122 & 1 & 105694.6 & .001 & b^{4} F_{5 / 2}-y^{4} F_{7 / 2}^{0}\end{array}$

$\begin{array}{lllll}944.605 & 0 & 105864.4 & .011 & c^{2} D_{3 / 2}-w^{2} P_{3 / 2}^{0}\end{array}$

$\begin{array}{lllll}943.156 & 1 \mathrm{H} & 106027.0 & .000 & a^{4} P_{5 / 2}-x^{2} D_{5 / 2}^{0}\end{array}$

$\begin{array}{lllll}941.917 & 10 & 106166.5 & .001 & a^{4} F_{9 / 2}-z^{2} G_{9 / 2}^{0}\end{array}$ 
Table I. Continued

Wavelength

(A)

\subsection{1}

937.710

937.046

936.730

935.852

935.665

934.934

933.623

933.522

932.852

932.245

929.735

928.473

928.407

926.909

926.303

926.121

925.299

924.299

922.600

922.173

921.164

921.035

920.659

920.408

920.147

918.946

918.627

918.386

918.088

916.258

915.656

914.360

913.144

910.050

909.127

908.219

905.180
904.584

903.107

900.745

899.993

897.728

897.207

896.534

896.061

895.743

$895.602 \quad 400$

895.451

894.610

894.323

893.618

891.192

890.197

889.776

888.314

886.045

885.863

885.474

884.991

881.078

880.799

880.351

877.310

Int.

350

350

500

100

50

200

500

500

300

30

500

400

100

400

100

50

350

50

250

500

50

450

350

300

250

150

200

350

25

500

50

500

200

500

15

000

200

25

50

400

200

500

10

500

250

200

15

150

500

500

100

100

150

400

10

250

250

30

50

$\left(\mathrm{cm}^{-2}\right)$

106382

106382.9

106718.3

106754.3

106854.5

106875.9

106959.4

107109.6

107121.2

107198.1

107267.9

107557.5

107703.7

107711.4

107885.5

107956.0

107977.3

108073.2

108190.1

108389.3

108439.5

108558.3

108573.5

108617.8

108647.5

108678.3

108820.3

108858.1

108886.7

108922.0

109139.6

109211.3

109366.1

109511.8

109884.1

109995.6

110105.6

110475.3

110548.1

110728.9

111019.2

111112.0

111392.3

111457.0

111540.7

111599.5

111639.2

111656.7

111675.6

111780.6

111816.4

111904.6

112209.3

112334.7

112387.8

112572.8

112861.1

112884.3

112933.9

112995.5

113497.3

113533.3

113591.1

113984.8
Table I. Continued

Wavelength Int. Wavenum ber O-C Classification
( $\AA)$

$\left(\mathrm{cm}^{-1}\right)$

(A)

(A)

$876.897 \quad 300$

$\begin{aligned} .002 & a^{2} G_{7 / 2}-y^{2} F_{7 / 2}^{0} \\ -.002 & a^{2} H_{9 / 2}-z^{2} H_{1 / 2}^{0} \\ .000 & a^{4} P_{5 / 2}-x^{2} F_{7 / 2}^{0} \\ .000 & a^{4} F_{3 / 2}-z^{2} F_{5 / 2}^{0} \\ -.004 & a^{4} F_{9 / 2}-z^{4} D_{7 / 2}^{0} \\ .004 & a^{4} F_{7 / 2}-y^{2} D_{5 / 2}^{0} \\ .004 & c^{2} D_{5 / 2}-v^{2} F_{5 / 2}^{0}\end{aligned}$

$875.661 \quad 500$

114038.5

114057.9

114199.4

$-.001 \quad b^{2} F_{s / 2}-v^{2} F_{s / 2}^{0}$

$875.223 \quad 500 \quad 114256.6$

$873.825 \quad 10$

114439.4

$872.109 \quad 10 \quad 114664.6$

$871.741 \quad 50 \quad 114713.0$

$.001 \quad b^{4} F_{5 / 2}-x^{2} P_{3 / 2}^{0}$

$\begin{cases}-.006 & c^{2} D_{3 / 2}-v^{2} D_{3 / 2}^{0}\end{cases}$

$-.002 \quad a^{4} F_{5 / 2}-y^{2} F_{5 / 2}^{0}$

$-.005 \quad b^{4} F_{9 / 2}-y^{4} F_{9 / 2}^{0}$

$.002 \quad a^{4} P_{1 / 2}-x^{2} D_{3 / 2}^{0}$

$.001 \quad a^{2} D_{3 / 2}-y^{2} P_{3 / 2}^{0}$

$-.003 \quad a^{2} H_{9 / 2}-x^{2} F_{7 / 2}^{0}$

$.005 \quad a^{2} P_{1 / 2}-y^{2} P_{3 / 2}^{0}$

$.001 \quad a^{2} F_{5 / 2}-x^{4} D_{3 / 2}^{0}$

$.000 \quad a^{2} G_{7 / 2}-x^{2} D_{s / 2}^{0}$

$.004 \quad a^{4} F_{5 / 2}-y^{4} D_{7 / 2}^{0}$

$.002 \quad b^{4} P_{3 / 2}-w^{2} F_{5 / 2}^{0}$

$.002 \quad a^{2} P_{3 / 2}-x^{2} P_{1 / 2}^{0}$

$-.003 \quad b^{2} F_{5 / 2}-w^{2} P_{3 / 2}^{0}$

$.001 \quad b^{4} F_{5 / 2}-w^{2} D_{5 / 2}^{0}$

$-.001 \quad a^{2} D_{3 / 2}-x^{2} F_{5 / 2}^{0}$

$-.004 \quad a^{4} F_{7 / 2}-z^{4} P_{5 / 2}^{0}$

$-.002 \quad a^{2} P_{3 / 2}^{7 / 2}-y^{4} F_{5 / 2}^{0}$

$\begin{cases}-.002 & b^{2} F_{5 / 2}-w^{2} F_{7 / 2}^{0} \\ -.002 & a^{2} G_{7 / 2}-x^{2} F_{7 / 2}^{0}\end{cases}$

$\begin{aligned}-.002 & a^{2} G_{7 / 2}-x^{2} F_{7 / 2}^{0} \\ .000 & a^{4} F_{7 / 2}-z^{2} G_{9 / 2}^{0}\end{aligned}$

$.001 \quad a^{4} P_{3 / 2}^{7 / 2}-x^{2} D_{3 / 2}^{0}$

$.002 \quad a^{4} P_{3 / 2}-z^{2} D_{5 / 2}^{0}$

$.001 \quad a^{4} P_{5 / 2}-y^{2} P_{3 / 2}^{0}$

$.003 \quad a^{4} F_{3 / 2}-y^{4} D_{3 / 2}^{0}$

$-.002 \quad b^{2} P_{1 / 2}-v^{2} P_{1 / 2}^{0}$

$.003 \quad a^{2} P_{3 / 2}-x^{4} D_{1 / 2}^{0}$

$-.003 \quad a^{4} F_{7 / 2}^{3 / 2}-z^{4} D_{7 / 2}^{0}$

$-.006 \quad b^{4} F_{3 / 2}-x^{2} P_{3 / 2}^{0}$

$-.014 \quad a^{4} F_{3 / 2}-z^{4} S_{3 / 2}^{0}$

$.000 \quad a^{4} F_{5 / 2}-z^{4} P_{3 / 2}^{0}$

$-.004 \quad b^{2} P_{3 / 2}-v^{2} P_{3 / 2}^{0}$

$.002 \quad b^{4} F_{5 / 2}-y^{4} P_{3 / 2}^{0}$

$-.007 \quad b^{2} F_{7 / 2}-v^{2} F_{5 / 2}^{0}$

$-.002 \quad b^{4} F_{7 / 2}-y^{4} P_{5 / 2}^{0}$

$-.001 \quad a^{2} D_{5 / 2}^{7 / 2}-y^{4} F_{5 / 2}^{0}$

$-.001 \quad b^{4} F_{5 / 2}-x^{4} D_{7 / 2}^{0}$

$.000 \quad b^{4} F_{7 / 2}-y^{4} F_{9 / 2}^{0}$

$.001 \quad a^{4} P_{3 / 2}-y^{2} P_{1 / 2}^{0}$

$-.002 \quad b^{2} G_{7 / 2}-v^{2} D_{5 / 2}^{0}$

$.000 \quad a^{2} D_{3 / 2}-y^{4} F_{3 / 2}^{0}$

$-.006 \quad b^{4} P_{5 / 2}-w^{2} P_{3 / 2}^{0}$

$-.002 \quad b^{4} F_{5 / 2}-w^{2} D_{3 / 2}^{0}$

$.000 \quad c^{2} D_{3 / 2}-v^{2} D_{3 / 2}^{0}$

$-.003 \quad a^{4} F_{5 / 2}-z^{4} P_{5 / 2}^{0}$

$-.001 \quad a^{2} G_{7 / 2}-x^{2} F_{s / 2}^{0}$

$.002 \quad a^{2} F_{7 / 2}-x^{4} D_{5 / 2}^{0}$

$-.002 \quad b^{4} P_{1 / 2}-w^{2} P_{3 / 2}^{0}$

$-.002 \quad a^{4} F_{7 / 2}^{1 / 2}-z^{2} D_{5 / 2}^{0}$

$-.001 \quad a^{4} F_{9 / 2}-y^{2} F_{7 / 2}^{0}$

$.001 \quad a^{4} F_{5 / 2}-z^{4} D_{7 / 2}^{0 / 2}$

$.001 \quad a^{4} F_{3 / 2}-z^{4} P_{1 / 2}^{0}$

$.001 \quad a^{4} F_{5 / 2}-z^{2} P_{3 / 2}^{0}$

$-.001 \quad b^{2} G_{7 / 2}-v^{2} F_{7 / 2}^{0}$

$.006 \quad a^{4} P_{3 / 2}-x^{2} D_{5 / 2}^{0}$

$.000 \quad c^{2} D_{s / 2}-v^{2} D_{s / 2}^{0}$

$-.001 \quad a^{4} F_{3 / 2}-z^{4} P_{3 / 2}^{0}$

$.000 \quad a^{4} F_{9 / 2}-z^{2} H_{11 / 2}^{0}$

$-.002 \quad b^{2} D_{3 / 2}^{9 / 2}-w^{2} P_{3 / 2}^{0}$

871.387

870.393

870.325

869.616

868.371

867.171

865.769

865.490

865.083

863.138

50

10

300

114713.0
114759.6

114890.6

100

50

114899.6 
Table II. Even levels of triply ionized tungsten (WIV). The level, level uncertainty, and $O-C$ values are given in units of $\mathrm{cm}^{-1}$. The number of transitions to each level is given in the column headed "No."

\begin{tabular}{|c|c|c|c|c|c|c|c|c|c|}
\hline Config. & Term & $J$ & Level & Unc. & No. & $\mathrm{O}-\mathrm{C}$ & \multicolumn{3}{|c|}{ Leading Percentages } \\
\hline $5 d^{3}$ & $a^{4} F$ & $\begin{array}{l}3 / 2 \\
5 / 2 \\
7 / 2 \\
9 / 2\end{array}$ & $\begin{array}{r}.00 \\
3537.49 \\
6744.63 \\
9256.39\end{array}$ & $\begin{array}{l}.15 \\
.13 \\
.12 \\
.16\end{array}$ & $\begin{array}{l}18 \\
21 \\
18 \\
12\end{array}$ & $\begin{array}{r}-38 \\
--76 \\
-50 \\
18\end{array}$ & $\begin{array}{l}86 \\
96 \\
92 \\
71\end{array}$ & $23^{2} G$ & \\
\hline $5 d^{3}$ & $a^{4} P$ & $\begin{array}{l}1 / 2 \\
3 / 2 \\
5 / 2\end{array}$ & $\begin{array}{l}11772.92 \\
10220.65 \\
17189.88\end{array}$ & $\begin{array}{l}.14 \\
.12 \\
.09\end{array}$ & $\begin{array}{l}12 \\
19 \\
24\end{array}$ & $\begin{array}{r}8 \\
88 \\
109\end{array}$ & $\begin{array}{l}58 \\
40 \\
89\end{array}$ & $\begin{array}{l}40^{2} P \\
39^{2} P\end{array}$ & $8^{2} D 2$ \\
\hline $5 d^{3}$ & $a^{2} G$ & $\begin{array}{l}7 / 2 \\
9 / 2\end{array}$ & $\begin{array}{l}15260.92 \\
24096.03\end{array}$ & $\begin{array}{l}.10 \\
.12\end{array}$ & $\begin{array}{l}22 \\
14\end{array}$ & $\begin{array}{r}9 \\
26\end{array}$ & $\begin{array}{l}88 \\
51\end{array}$ & $42{ }^{2} H$ & \\
\hline $5 d^{3}$ & $a^{2} H$ & $\begin{array}{r}9 / 2 \\
11 / 2\end{array}$ & $\begin{array}{l}16204.85 \\
22223.06\end{array}$ & $\begin{array}{l}.13 \\
.16\end{array}$ & $\begin{array}{r}16 \\
7\end{array}$ & $\begin{array}{r}-21 \\
1\end{array}$ & $\begin{array}{r}53 \\
100\end{array}$ & $24{ }^{2} G$ & $22^{4} \mathrm{~F}$ \\
\hline $5 d^{3}$ & $a^{2} P$ & $\begin{array}{l}1 / 2 \\
3 / 2\end{array}$ & $\begin{array}{l}18364.76 \\
25167.81\end{array}$ & $\begin{array}{l}.15 \\
.08\end{array}$ & $\begin{array}{l}15 \\
26\end{array}$ & $\begin{array}{l}-67 \\
-13\end{array}$ & $\begin{array}{l}54 \\
48\end{array}$ & $\begin{array}{l}42{ }^{4} P \\
20{ }^{2} D 2\end{array}$ & $13^{2} D 1$ \\
\hline $5 d^{3}$ & $a^{2} D 2$ & $\begin{array}{l}3 / 2 \\
5 / 2\end{array}$ & $\begin{array}{l}18519.01 \\
22766.37\end{array}$ & $\begin{array}{l}.09 \\
.07\end{array}$ & $\begin{array}{l}25 \\
32\end{array}$ & $\begin{array}{l}17 \\
70\end{array}$ & $\begin{array}{l}30 \\
73\end{array}$ & $\begin{array}{l}47{ }^{4} P \\
10^{2} D 1\end{array}$ & $10^{2} \mathrm{D} 1$ \\
\hline $5 d^{3}$ & $a^{2} F$ & $\begin{array}{l}5 / 2 \\
7 / 2\end{array}$ & $\begin{array}{l}27937.63 \\
28412.13\end{array}$ & $\begin{array}{l}.07 \\
.08\end{array}$ & $\begin{array}{l}28 \\
26\end{array}$ & $\begin{array}{r}-4 \\
22\end{array}$ & $\begin{array}{l}80 \\
91\end{array}$ & $9\left({ }^{3} F\right)^{2} F$ & \\
\hline $5 d^{3}$ & $b^{2} D 1$ & $\begin{array}{l}5 / 2 \\
3 / 2\end{array}$ & $\begin{array}{l}43286.60 \\
43741.52\end{array}$ & $\begin{array}{l}.06 \\
.07\end{array}$ & $\begin{array}{l}26 \\
18\end{array}$ & $\begin{array}{r}104 \\
-49\end{array}$ & $\begin{array}{l}74 \\
68\end{array}$ & $\begin{array}{l}10^{2} D 2 \\
30^{2} D 2\end{array}$ & \\
\hline $5 d^{2}\left({ }^{3} F\right) 6 s$ & $b^{4} F$ & $\begin{array}{l}3 / 2 \\
5 / 2 \\
7 / 2 \\
9 / 2\end{array}$ & $\begin{array}{l}32692.40 \\
35093.78 \\
39029.73 \\
43224.40\end{array}$ & $\begin{array}{l}.06 \\
.06 \\
.05 \\
.07\end{array}$ & $\begin{array}{l}25 \\
32 \\
30 \\
18\end{array}$ & $\begin{array}{r}47 \\
16 \\
-50 \\
-48\end{array}$ & $\begin{array}{l}94 \\
89 \\
97 \\
95\end{array}$ & & \\
\hline $5 d^{2}\left({ }^{3} P\right) 6 s$ & $b^{4} P$ & $\begin{array}{l}1 / 2 \\
3 / 2 \\
5 / 2\end{array}$ & $\begin{array}{l}45516.94 \\
47679.55 \\
46087.55\end{array}$ & $\begin{array}{l}.07 \\
.04 \\
.04\end{array}$ & $\begin{array}{l}17 \\
32 \\
34\end{array}$ & $\begin{array}{r}50 \\
7 \\
-73\end{array}$ & $\begin{array}{l}95 \\
90 \\
27\end{array}$ & $30\left({ }^{1} D\right)^{2} D$ & $26\left({ }^{3} F\right)^{2} F$ \\
\hline $5 d^{2}\left({ }^{3} F\right) 6 s$ & $b^{2} F$ & $\begin{array}{l}5 / 2 \\
7 / 2\end{array}$ & $\begin{array}{l}49337.04 \\
52828.24\end{array}$ & $\begin{array}{l}.03 \\
.04\end{array}$ & $\begin{array}{l}39 \\
29\end{array}$ & $\begin{array}{r}3 \\
34\end{array}$ & $\begin{array}{l}46 \\
63\end{array}$ & $\begin{array}{l}41\left({ }^{3} P\right)^{4} P \\
32\left({ }^{1} G\right)^{2} G\end{array}$ & \\
\hline $5 d^{2}\left({ }^{1} D\right) 6 s$ & $c^{2} D$ & $\begin{array}{l}3 / 2 \\
5 / 2\end{array}$ & $\begin{array}{l}51860.40 \\
56415.54\end{array}$ & $\begin{array}{l}.04 \\
.04\end{array}$ & $\begin{array}{l}30 \\
32\end{array}$ & $\begin{array}{r}-47 \\
75\end{array}$ & $\begin{array}{l}73 \\
58\end{array}$ & $\begin{array}{l}10\left({ }^{3} P\right)^{2} P \\
32\left({ }^{3} P\right)^{4} P\end{array}$ & \\
\hline $5 d^{2}\left({ }^{1} G\right) 6 s$ & $b^{2} G$ & $\begin{array}{l}9 / 2 \\
7 / 2\end{array}$ & $\begin{array}{l}57050.05 \\
58372.54\end{array}$ & $\begin{array}{l}.05 \\
.04\end{array}$ & $\begin{array}{l}16 \\
23\end{array}$ & $\begin{array}{r}-69 \\
52\end{array}$ & $\begin{array}{l}94 \\
66\end{array}$ & $30\left({ }^{3} F\right)^{2} F$ & \\
\hline $5 d^{2}\left({ }^{3} P\right) 6 s$ & $b^{2} P$ & $\begin{array}{l}1 / 2 \\
3 / 2\end{array}$ & $\begin{array}{l}59628.18 \\
64680.96\end{array}$ & $\begin{array}{l}.04 \\
.04\end{array}$ & $\begin{array}{l}14 \\
19\end{array}$ & $\begin{array}{l}-39 \\
-21\end{array}$ & $\begin{array}{l}88 \\
84\end{array}$ & & \\
\hline $5 d^{2}\left({ }^{1} S\right) 6 s$ & $a^{2} S$ & $1 / 2$ & 77607.89 & .06 & 9 & -39 & 92 & & \\
\hline $5 d 6 s^{2}$ & $d^{2} D$ & $\begin{array}{l}3 / 2 \\
5 / 2\end{array}$ & $\begin{array}{l}87172.17 \\
94725.05\end{array}$ & $\begin{array}{l}.08 \\
.10\end{array}$ & $\begin{array}{l}6 \\
5\end{array}$ & & & & \\
\hline
\end{tabular}


182 L. Iglesias, V. Kaufman, O. Garcia-Riquelme and F. R. Rico

Table III. Odd levels of triply ionized tungsten (WIV). The level and level uncertainty values and the $O$-C are given in units of $\mathrm{cm}^{-1}$. The number of transitions from each level is given in the column headed "No."

\begin{tabular}{|c|c|c|c|c|c|c|c|c|c|}
\hline Config. & Term & $J$ & Level & Unc. & No. & $\mathrm{O}-\mathrm{C}$ & Leadi & Percentages & \\
\hline $5 d^{2}\left({ }^{3} F\right) 6 p$ & $z^{4} G$ & $\begin{array}{r}5 / 2 \\
7 / 2 \\
9 / 2 \\
11 / 2\end{array}$ & $\begin{array}{r}85289.99 \\
92845.71 \\
99028.26 \\
109190.30\end{array}$ & $\begin{array}{l}.05 \\
.05 \\
.06 \\
.17\end{array}$ & $\begin{array}{r}13 \\
15 \\
12 \\
5\end{array}$ & $\begin{array}{r}-333 \\
-55 \\
185 \\
37\end{array}$ & $\begin{array}{l}64 \\
77 \\
51 \\
94\end{array}$ & $\begin{array}{l}19\left({ }^{3} F\right)^{2} F \\
10\left({ }^{3} F\right)^{2} F \\
19\left({ }^{3} F\right)^{4} F\end{array}$ & $\begin{array}{l}11\left({ }^{1} D\right)^{2} F \\
16\left({ }^{3} F\right)^{2} G\end{array}$ \\
\hline $5 d^{2}\left({ }^{3} F\right) 6 p$ & $z^{4} F$ & $\begin{array}{l}3 / 2 \\
5 / 2 \\
7 / 2 \\
9 / 2\end{array}$ & $\begin{array}{r}89893.06 \\
94525.28 \\
100322.36 \\
105263.40\end{array}$ & $\begin{array}{l}.04 \\
.05 \\
.06 \\
.06\end{array}$ & $\begin{array}{l}16 \\
17 \\
17 \\
12\end{array}$ & $\begin{array}{r}464 \\
-255 \\
8 \\
0\end{array}$ & $\begin{array}{l}49 \\
42 \\
43 \\
19\end{array}$ & $\begin{array}{l}33\left({ }^{3} F\right)^{2} D \\
21\left({ }^{3} F\right)^{2} D \\
22\left({ }^{3} F\right)^{4} D \\
49\left({ }^{3} F\right)^{4} G\end{array}$ & $\begin{array}{l}17\left({ }^{3} F\right)^{4} D \\
10\left({ }^{3} F\right)^{2} F \\
16\left({ }^{3} F\right)^{2} G\end{array}$ \\
\hline $5 d^{2}\left({ }^{3} P\right) 6 p$ & $z^{4} D$ & $\begin{array}{l}1 / 2 \\
3 / 2 \\
5 / 2 \\
7 / 2\end{array}$ & $\begin{array}{r}98463.71 \\
103952.54 \\
98691.76 \\
116110.43\end{array}$ & $\begin{array}{l}.06 \\
.05 \\
.05 \\
.07\end{array}$ & $\begin{array}{l}10 \\
19 \\
16 \\
18\end{array}$ & $\begin{array}{r}264 \\
-8 \\
-215 \\
355\end{array}$ & $\begin{array}{l}43 \\
40 \\
14 \\
40\end{array}$ & $\begin{array}{l}21\left({ }^{3} P\right)^{2} S \\
17\left({ }^{3} P\right)^{4} S \\
30\left(\left(^{3} F\right)^{4} F\right. \\
15\left({ }^{3} F\right)^{2} F\end{array}$ & $\begin{array}{l}11\left({ }^{3} F\right)^{4} D \\
11\left({ }^{3} P\right)^{2} P \\
23\left({ }^{3} F\right)^{4} G \\
12\left({ }^{1} D\right)^{2} F\end{array}$ \\
\hline $5 d^{2}\left({ }^{1} D\right) 6 p$ & $z^{2} D$ & $\begin{array}{l}3 / 2 \\
5 / 2\end{array}$ & $\begin{array}{r}99867.18 \\
119079.04\end{array}$ & $\begin{array}{l}.04 \\
.09\end{array}$ & $\begin{array}{l}18 \\
18\end{array}$ & $\begin{array}{l}-379 \\
-430\end{array}$ & $\begin{array}{l}27 \\
37\end{array}$ & $\begin{array}{l}19\left({ }^{3} P\right)^{4} D \\
30\left({ }^{3} P\right)^{4} P\end{array}$ & $\begin{array}{l}17\left({ }^{3} F\right)^{2} D \\
13\left({ }^{3} P\right)^{2} D\end{array}$ \\
\hline $5 d^{2}\left({ }^{3} F\right) 6 p$ & $y^{2} D$ & $\begin{array}{l}3 / 2 \\
5 / 2\end{array}$ & $\begin{array}{l}100449.92 \\
113620.96\end{array}$ & $\begin{array}{l}.06 \\
.07\end{array}$ & $\begin{array}{l}19 \\
15\end{array}$ & $\begin{array}{r}-252 \\
102\end{array}$ & $\begin{array}{l}15 \\
31\end{array}$ & $\begin{array}{l}36\left({ }^{3} F\right)^{4} F \\
25\left({ }^{3} P\right)^{4} D\end{array}$ & $\begin{array}{l}24\left({ }^{3} F\right)^{4} D \\
18\left({ }^{3} F\right)^{4} D\end{array}$ \\
\hline $5 d^{2}\left({ }^{3} P\right) 6 p$ & $z^{2} S$ & $1 / 2$ & 101275.42 & .06 & 9 & -99 & 39 & $27\left({ }^{3} P\right)^{4} P$ & $22\left({ }^{3} P\right)^{4} D$ \\
\hline $5 d^{2}\left({ }^{3} F\right) 6 p$ & $z^{2} F$ & $\begin{array}{l}7 / 2 \\
5 / 2\end{array}$ & $\begin{array}{l}102867.63 \\
106754.40\end{array}$ & $\begin{array}{l}.06 \\
.06\end{array}$ & $\begin{array}{l}17 \\
20\end{array}$ & $\begin{array}{r}152 \\
-283\end{array}$ & $\begin{array}{l}26 \\
32\end{array}$ & $\begin{array}{l}26\left({ }^{3} F\right)^{2} G \\
17\left({ }^{3} F\right)^{4} D\end{array}$ & $\begin{array}{l}23\left({ }^{3} F\right)^{4} D \\
12\left({ }^{3} F\right)^{2} D\end{array}$ \\
\hline $5 d^{2}\left({ }^{3} F\right) 6 p$ & $y^{4} D$ & $\begin{array}{l}1 / 2 \\
3 / 2 \\
5 / 2 \\
7 / 2\end{array}$ & $\begin{array}{l}103682.37 \\
108922.34 \\
103216.51 \\
111515.16\end{array}$ & $\begin{array}{l}.08 \\
.07 \\
.05 \\
.07\end{array}$ & $\begin{array}{r}8 \\
16 \\
17 \\
17\end{array}$ & $\begin{array}{r}-201 \\
-19 \\
326 \\
-211\end{array}$ & $\begin{array}{l}62 \\
53 \\
24 \\
32\end{array}$ & $\begin{array}{l}22\left({ }^{3} P\right)^{4} D \\
22\left({ }^{3} F\right)^{2} D \\
18\left({ }^{1} D\right)^{2} F \\
33\left({ }^{1} G\right)^{2} G\end{array}$ & $\begin{array}{l}14\left({ }^{3} P\right)^{4} D \\
17\left({ }^{3} F\right)^{2} F \\
16\left({ }^{3} F\right)^{2} G\end{array}$ \\
\hline $5 d^{2}\left({ }^{3} F\right) 6 p$ & $z^{2} G$ & $\begin{array}{l}7 / 2 \\
9 / 2\end{array}$ & $\begin{array}{l}106730.40 \\
115422.93\end{array}$ & $\begin{array}{l}.06 \\
.10\end{array}$ & $\begin{array}{l}16 \\
11\end{array}$ & $\begin{array}{r}-165 \\
241\end{array}$ & $\begin{array}{l}29 \\
67\end{array}$ & $\begin{array}{l}38\left({ }^{3} F\right)^{4} F \\
20\left({ }^{3} F\right)^{4} F\end{array}$ & $15\left({ }^{1} G\right)^{2} G$ \\
\hline $5 d^{2}\left({ }^{1} G\right) 6 p$ & $y^{2} G$ & $\begin{array}{l}7 / 2 \\
9 / 2\end{array}$ & $\begin{array}{l}108871.96 \\
119694.03\end{array}$ & $\begin{array}{l}.07 \\
.12\end{array}$ & $\begin{array}{l}16 \\
10\end{array}$ & $\begin{array}{r}-238 \\
-32\end{array}$ & $\begin{array}{l}15 \\
57\end{array}$ & $\begin{array}{l}29\left({ }^{1} D\right)^{2} F \\
37\left({ }^{1} G\right)^{2} H\end{array}$ & $20\left({ }^{1} G\right)^{2} F$ \\
\hline $5 d^{2}\left({ }^{3} P\right) 6 p$ & $z^{4} S$ & $3 / 2$ & 109882.34 & .07 & 14 & -180 & 43 & $19\left({ }^{1} D\right)^{2} D$ & $11\left({ }^{3} P\right)^{4} P$ \\
\hline $5 d^{2}\left({ }^{1} G\right) 6 p$ & $z^{2} H$ & $\begin{array}{r}9 / 2 \\
11 / 2\end{array}$ & $\begin{array}{l}110252.75 \\
122847.40\end{array}$ & $\begin{array}{l}.08 \\
.20\end{array}$ & $\begin{array}{r}11 \\
6\end{array}$ & $\begin{array}{l}227 \\
460\end{array}$ & $\begin{array}{l}48 \\
94\end{array}$ & $37\left({ }^{3} F\right)^{4} F$ & $14\left({ }^{1} G\right)^{2} G$ \\
\hline $5 d^{2}\left({ }^{1} D\right) 6 p$ & $y^{2} F$ & $\begin{array}{l}5 / 2 \\
7 / 2\end{array}$ & $\begin{array}{l}110658.47 \\
121644.05\end{array}$ & $\begin{array}{l}.06 \\
.10\end{array}$ & $\begin{array}{l}16 \\
12\end{array}$ & $\begin{array}{r}375 \\
-47\end{array}$ & $\begin{array}{l}37 \\
45\end{array}$ & $\begin{array}{l}19\left({ }^{3} P\right)^{4} D \\
41\left({ }^{3} P\right)^{4} D\end{array}$ & $15\left({ }^{3} F\right)^{4} D$ \\
\hline $5 d^{2}\left({ }^{3} P\right) 6 p$ & $z^{4} P$ & $\begin{array}{l}1 / 2 \\
3 / 2 \\
5 / 2\end{array}$ & $\begin{array}{l}112861.20 \\
113533.11 \\
115317.71\end{array}$ & $\begin{array}{l}.10 \\
.09 \\
.09\end{array}$ & $\begin{array}{r}7 \\
12 \\
19\end{array}$ & $\begin{array}{r}49 \\
366 \\
243\end{array}$ & $\begin{array}{l}41 \\
47 \\
28\end{array}$ & $\begin{array}{l}31\left({ }^{1} D\right)^{2} P \\
17\left({ }^{1} D\right)^{2} P \\
31\left({ }^{1} D\right)^{2} D\end{array}$ & $\begin{array}{c}16\left({ }^{3} P\right)^{2} S \\
9\left({ }^{3} P\right)^{4} S \\
25\left({ }^{3} P\right)^{4} D\end{array}$ \\
\hline $5 d^{2}\left({ }^{1} D\right) 6 p$ & $z^{2} P$ & $\begin{array}{l}3 / 2 \\
1 / 2\end{array}$ & $\begin{array}{l}116421.86 \\
117725.20\end{array}$ & $\begin{array}{l}.07 \\
.07\end{array}$ & $\begin{array}{l}13 \\
10\end{array}$ & $\begin{array}{r}688 \\
-104\end{array}$ & $\begin{array}{l}25 \\
40\end{array}$ & $\begin{array}{l}24\left({ }^{3} P\right)^{4} P \\
24\left({ }^{3} P\right)^{4} P\end{array}$ & $\begin{array}{l}19\left({ }^{1} D\right)^{2} D \\
16\left({ }^{3} P\right)^{2} S\end{array}$ \\
\hline $5 d^{2}\left({ }^{3} P\right) 6 p$ & $x^{2} D$ & $\begin{array}{l}3 / 2 \\
5 / 2\end{array}$ & $\begin{array}{l}119041.11 \\
123216.93\end{array}$ & $\begin{array}{l}.10 \\
.08\end{array}$ & $\begin{array}{l}14 \\
20\end{array}$ & $\begin{array}{r}-420 \\
564\end{array}$ & $\begin{array}{l}50 \\
43\end{array}$ & $\begin{array}{l}21\left({ }^{3} P\right)^{2} P \\
26\left({ }^{3} P\right)^{4} P\end{array}$ & $\begin{array}{c}9\left({ }^{3} P\right)^{4} S \\
13\left({ }^{1} G\right)^{2} F\end{array}$ \\
\hline $5 d^{2}\left({ }^{3} P\right) 6 p$ & $y^{2} P$ & $\begin{array}{l}1 / 2 \\
3 / 2\end{array}$ & $\begin{array}{l}121677.79 \\
126076.70\end{array}$ & $\begin{array}{l}.09 \\
.11\end{array}$ & $\begin{array}{r}9 \\
16\end{array}$ & $\begin{array}{r}-96 \\
-417\end{array}$ & $\begin{array}{l}57 \\
48\end{array}$ & $\begin{array}{l}20\left({ }^{1} S\right)^{2} P \\
17\left({ }^{3} P\right)^{2} D\end{array}$ & $\begin{array}{l}12\left({ }^{3} P\right)^{4} D \\
12\left({ }^{3} D\right)^{2} D\end{array}$ \\
\hline $5 d^{2}\left({ }^{1} G\right) 6 p$ & $x^{2} F$ & $\begin{array}{l}7 / 2 \\
5 / 2\end{array}$ & $\begin{array}{l}123908.20 \\
127077.24\end{array}$ & $\begin{array}{l}.09 \\
.07\end{array}$ & $\begin{array}{l}15 \\
16\end{array}$ & $\begin{array}{r}-562 \\
-88\end{array}$ & $\begin{array}{l}60 \\
79\end{array}$ & $\begin{array}{c}24\left({ }^{1} G\right)^{2} G \\
8\left({ }^{3} F\right)^{2} D\end{array}$ & \\
\hline $5 d^{2}\left({ }^{1} S\right) 6 p$ & $x^{2} P$ & $\begin{array}{l}1 / 2 \\
3 / 2\end{array}$ & $\begin{array}{l}133358.14 \\
142203.49\end{array}$ & $\begin{array}{l}.09 \\
.10\end{array}$ & $\begin{array}{r}9 \\
13\end{array}$ & $\begin{array}{r}139 \\
-74\end{array}$ & $\begin{array}{l}68 \\
86\end{array}$ & $21\left({ }^{3} P\right)^{2} P$ & \\
\hline $5 d\left({ }^{2} D\right) 6 s 6 p\left({ }^{3} P\right)$ & $y^{4} F$ & $\begin{array}{l}3 / 2 \\
5 / 2 \\
7 / 2 \\
9 / 2\end{array}$ & $\begin{array}{l}130118.60 \\
133785.47 \\
140788.56 \\
150422.0\end{array}$ & $\begin{array}{l}.17 \\
.18 \\
.11 \\
.4\end{array}$ & $\begin{array}{r}6 \\
11 \\
8 \\
3\end{array}$ & $\begin{array}{r}-333 \\
49 \\
118 \\
-19\end{array}$ & $\begin{array}{r}79 \\
71 \\
73 \\
100\end{array}$ & $\begin{array}{l}7\left({ }^{3} P\right)^{2} D \\
10\left({ }^{3} P\right)^{2} D \\
21{ }^{4} D\end{array}$ & \\
\hline $5 d\left({ }^{2} D\right) 6 s 6 p\left({ }^{3} P\right)$ & $x^{4} D$ & $\begin{array}{l}1 / 2 \\
3 / 2 \\
5 / 2 \\
7 / 2\end{array}$ & $\begin{array}{l}134379.44 \\
135823.21 \\
140317.03 \\
146205.65\end{array}$ & $\begin{array}{l}.23 \\
.29 \\
.16 \\
.25\end{array}$ & $\begin{array}{l}6 \\
3 \\
9 \\
6\end{array}$ & $\begin{array}{r}295 \\
-56 \\
-204 \\
-109\end{array}$ & $\begin{array}{l}82 \\
67 \\
59 \\
64\end{array}$ & $\begin{array}{l}11{ }^{4} P \\
25{ }^{4} P \\
23{ }^{4} F\end{array}$ & $8\left({ }^{3} P\right)^{2} P$ \\
\hline $5 d\left(\left(^{2} D\right) 6 s 6 p\left(\left(^{3} P\right)\right.\right.$ & $w^{2} D$ & $\begin{array}{l}5 / 2 \\
3 / 2\end{array}$ & $\begin{array}{l}143533.47 \\
146750.22\end{array}$ & $\begin{array}{l}.17 \\
.19\end{array}$ & $\begin{array}{r}10 \\
9\end{array}$ & $\begin{array}{r}-108 \\
102\end{array}$ & $\begin{array}{l}38 \\
58\end{array}$ & $\begin{array}{l}25{ }^{4} F \\
24{ }^{4} P\end{array}$ & $21^{4} D$ \\
\hline $5 d\left({ }^{2} D\right) 6 s 6 p\left({ }^{3} P\right)$ & $y^{4} P$ & $\begin{array}{l}1 / 2 \\
3 / 2 \\
5 / 2\end{array}$ & $\begin{array}{l}147405.37 \\
145569.33 \\
149758.30\end{array}$ & $\begin{array}{l}.37 \\
.18 \\
.22\end{array}$ & $\begin{array}{l}3 \\
7 \\
8\end{array}$ & $\begin{array}{r}167 \\
-71 \\
235\end{array}$ & $\begin{array}{l}92 \\
42 \\
36\end{array}$ & $\begin{array}{l}22{ }^{4} D \\
46\left({ }^{3} P\right)^{2} F\end{array}$ & $19\left({ }^{3} P\right)^{2} P$ \\
\hline
\end{tabular}


Table III. Continued

\begin{tabular}{|c|c|c|c|c|c|c|c|c|c|}
\hline Config. & Term & $J$ & Level & Unc. & No. & $\mathrm{O}-\mathrm{C}$ & Lead & ercentages & \\
\hline $5 d\left({ }^{2} D\right) 6 s 6 p\left({ }^{3} P\right)$ & $w^{2} F$ & $\begin{array}{l}5 / 2 \\
7 / 2\end{array}$ & $\begin{array}{l}155752.99 \\
157984.23\end{array}$ & $\begin{array}{l}.28 \\
.31\end{array}$ & $\begin{array}{l}4 \\
4\end{array}$ & $\begin{array}{r}99 \\
-94\end{array}$ & $\begin{array}{l}36 \\
84\end{array}$ & $26\left({ }^{3} P\right)^{2} D$ & $24{ }^{4} P$ \\
\hline $5 d\left({ }^{2} D\right) 6 s 6 p\left({ }^{3} P\right)$ & $w^{2} P$ & $\begin{array}{l}3 / 2 \\
1 / 2\end{array}$ & $\begin{array}{l}157726.00 \\
162651.8\end{array}$ & $\begin{array}{l}.21 \\
.7\end{array}$ & $\begin{array}{r}12 \\
1\end{array}$ & $\begin{array}{r}236 \\
8\end{array}$ & $\begin{array}{l}31 \\
73\end{array}$ & $\begin{array}{l}41\left({ }^{1} P\right)^{2} D \\
16\left({ }^{1} P\right)^{2} P\end{array}$ & $19^{4} P$ \\
\hline $5 d\left({ }^{2} D\right) 6 s 6 p\left({ }^{1} P\right)$ & $v^{2} F$ & $\begin{array}{l}5 / 2 \\
7 / 2\end{array}$ & $\begin{array}{l}163375.42 \\
171306.23\end{array}$ & $\begin{array}{l}.22 \\
.22\end{array}$ & $\begin{array}{r}10 \\
8\end{array}$ & $\begin{array}{r}109 \\
17\end{array}$ & $\begin{array}{l}86 \\
93\end{array}$ & & \\
\hline $5 d\left({ }^{2} D\right) 6 s 6 p\left({ }^{1} P\right)$ & $v^{2} D$ & $\begin{array}{l}3 / 2 \\
5 / 2\end{array}$ & $\begin{array}{l}163536.02 \\
169912.91\end{array}$ & $\begin{array}{l}.18 \\
.23\end{array}$ & $\begin{array}{r}11 \\
9\end{array}$ & $\begin{array}{r}-233 \\
67\end{array}$ & $\begin{array}{l}50 \\
79\end{array}$ & $\begin{array}{r}36\left({ }^{3} P\right)^{2} P \\
7\left({ }^{3} P\right)^{2} F\end{array}$ & \\
\hline $5 d\left({ }^{2} D\right) 6 s 6 p\left({ }^{1} P\right)$ & $v^{2} P$ & $\begin{array}{l}1 / 2 \\
3 / 2\end{array}$ & $\begin{array}{l}168767.50 \\
174786.10\end{array}$ & $\begin{array}{l}.27 \\
.20\end{array}$ & $\begin{array}{l}5 \\
7\end{array}$ & $\begin{array}{r}103 \\
-222\end{array}$ & $\begin{array}{l}79 \\
94\end{array}$ & $20\left({ }^{3} P\right)^{2} P$ & \\
\hline
\end{tabular}

Table IV. Least-squares fitted (LSF) and Hartree-Fock (HF) parameter values and their ratios for the $5 d^{3}+5 d^{2} 6 s$ configurations of $W^{+3}$

\begin{tabular}{|c|c|c|c|c|}
\hline \multirow[b]{2}{*}{ Config. } & \multirow[b]{2}{*}{ Parameter } & \multicolumn{2}{|l|}{ Value } & \multirow[b]{2}{*}{ LSF/HF } \\
\hline & & LSF & $\mathrm{HF}$ & \\
\hline $5 d^{3}$ & $\begin{array}{l}F^{2}(d d) \\
F^{4}(d d) \\
\zeta_{d} \\
\alpha \\
\beta\end{array}$ & $\begin{array}{r}47530(147) \\
29988(454) \\
2720(13) \\
25(\quad 4) \\
-90(57)\end{array}$ & $\begin{array}{r}60671 \\
40278 \\
2910\end{array}$ & $\begin{array}{l}0.78 \\
0.74 \\
0.93\end{array}$ \\
\hline $5 d^{2} 6 s$ & $\begin{array}{l}F^{2}(d d) \\
F^{4}(d d) \\
G^{2}(d s) \\
\zeta_{d} \\
\alpha \\
\beta\end{array}$ & $\begin{array}{r}48930(196) \\
28980(466) \\
17375(200) \\
2920(15) \\
40(4) \\
460(49)\end{array}$ & $\begin{array}{r}62828 \\
41882 \\
15719 \\
3129\end{array}$ & $\begin{array}{l}0.78 \\
0.69 \\
1.10 \\
0.93\end{array}$ \\
\hline C.I. & $R^{2}(d d, d s)$ & $21000(455)$ & 18438 & 1.14 \\
\hline
\end{tabular}

Table V. Least-squares fitted (LSF) and Hartree-Fock (HF) parameter values and their ratios for the $5 d^{2} 6 p+5 d 6 s 6 p$ configurations of $W^{+3}$

\begin{tabular}{|c|c|c|c|c|}
\hline \multirow[b]{2}{*}{ Config. } & \multirow[b]{2}{*}{ Parameter } & \multicolumn{2}{|l|}{ Value } & \multirow[b]{2}{*}{$\mathrm{LSF} / \mathrm{HF}$} \\
\hline & & LSF & $\mathrm{HF}$ & \\
\hline \multirow[t]{9}{*}{$5 d^{2} 6 p$} & $F^{2}(d d)$ & $48125(1030)$ & 63209 & 0.76 \\
\hline & $F^{4}(d d)$ & $27531(2600)$ & 42169 & 0.65 \\
\hline & $F^{2}(d p)$ & $18375(560)$ & 24906 & 0.74 \\
\hline & $G^{1}(d p)$ & $8625(225)$ & 9809 & 0.88 \\
\hline & $G^{3}(d p)$ & $2042(735)$ & 8556 & 0.24 \\
\hline & $\zeta_{d}$ & $3020(48)$ & 3163 & 0.95 \\
\hline & $\zeta_{p}$ & $8065(112)$ & 5124 & 1.51 \\
\hline & $\alpha$ & $65(22)$ & & \\
\hline & $\beta$ & $445(263)$ & & \\
\hline \multirow[t]{7}{*}{$5 d 6 s 6 p$} & $F^{2}(d p)$ & $22925(735)$ & 26393 & 0.87 \\
\hline & $G^{1}(s p)$ & $29340(312)$ & 45266 & 0.65 \\
\hline & $G^{2}(d s)$ & $20250(1020)$ & 15705 & 1.29 \\
\hline & $G^{1}(d p)$ & $9825(525)$ & 10051 & 0.97 \\
\hline & $G^{3}(d p)$ & $6533(980)$ & 8943 & 0.73 \\
\hline & $\zeta_{d}$ & $3250(76)$ & 3378 & 0.96 \\
\hline & $\zeta_{p}$ & $8850(174)$ & 5905 & 1.50 \\
\hline \multirow[t]{3}{*}{ C.I. } & $R^{1}(d p, p s)$ & $17940(2710)$ & 24351 & 0.74 \\
\hline & $R^{2}(d p, s p)$ & $12185(1990)$ & 15631 & 0.78 \\
\hline & $R^{2}(d d, d s)$ & $5460(4240)$ & 18075 & 0.30 \\
\hline
\end{tabular}

Table VI. Comparison of least-squares fit to Hartree-Fock $(L S F / H F)$ ratios for parameters of the known configurations in $W^{+3}$ and $T^{+3}$

\begin{tabular}{llllll}
\hline & \multicolumn{3}{l}{$\mathrm{W}^{+3}$} & & $\mathrm{Ta}^{+3}$ \\
\cline { 2 - 3 } \cline { 5 - 6 } Parameter & Config. & LSF/HF & & Config. & LSF $/ \mathrm{HF}^{2}$ \\
\hline$F^{2}(d d)$ & $5 d^{3}$ & 0.78 & & $5 d^{2}$ & 0.79 \\
$F^{4}(d d)$ & & 0.74 & & 0.75 \\
$\zeta_{d}$ & & 0.93 & & 0.92 \\
$G^{2}(d s)$ & $5 d^{2} 6 s$ & 1.10 & & $5 d 6 s$ & 1.05 \\
$\zeta_{d}$ & & 0.93 & & \\
$R^{2}(d d, d s)$ & C.I. & 1.14 & & C.I. & 0.89 \\
$F^{2}(d p)$ & $5 d^{2} 6 p$ & 0.74 & & $5 d 6 p$ & 0.78 \\
$G^{1}(d p)$ & & 0.88 & & 0.88 \\
$G^{3}(d p)$ & & 0.24 & & 0.40 \\
$\zeta_{d}$ & & 0.95 & & 0.95 \\
$\zeta_{p}$ & & 1.51 & & 1.53 \\
$G^{1}(s p)$ & $5 d 6 s 6 p$ & 0.65 & & $6 s 6 p$ & 0.64 \\
$\zeta_{p}$ & & 1.50 & & 1.45 \\
$R^{1}(d p, p s)$ & C.I. & 0.74 & C.I. & 0.56 \\
$R^{2}(d p, p s)$ & & 0.78 & & 0.66 \\
\hline
\end{tabular}

a Data taken from Ref. 1.

Table VII. Transition array of the doublet levels of the $5 d 6 s 6 p$ configuration with the ${ }^{2} D_{3 / 2,5 / 2}$ of the $5 d 6 s^{2}$ configuration

\begin{tabular}{rrll}
\hline & \multicolumn{3}{c}{$5 d\left({ }^{2} D\right) 6 s^{2}\left({ }^{1} S\right)^{2} D$} \\
& & $3 / 2$ & $5 / 2$ \\
\hline $5 d\left({ }^{2} D\right) 6 s 6 p\left({ }^{1} P\right)^{2} F$ & $5 / 2$ & 100 & - \\
& $7 / 2$ & & 100 \\
${ }^{2} D$ & $3 / 2$ & 50 & - \\
& $5 / 2$ & - & 100 \\
${ }^{2} P$ & $1 / 2$ & 100 & \\
& $3 / 2$ & - & 50 \\
$5 d\left({ }^{2} D\right) 6 s 6 p\left({ }^{3} P\right)^{2} F$ & $5 / 2$ & - & - \\
& $7 / 2$ & & - \\
${ }^{2} D$ & $3 / 2$ & - & - \\
& $5 / 2$ & - & - \\
${ }^{2} P$ & $1 / 2$ & - & - \\
& $3 / 2$ & - & - \\
\hline
\end{tabular}

